\title{
Mixed state entanglement measures as probe for confinement
}

\author{
Parul Jain ${ }^{1, *}$ and Subhash Mahapatra ${ }^{2, \dagger}$ \\ ${ }^{1}$ Department of Physics, Indian Institute of Technology, Bombay 400076, India \\ ${ }^{2}$ Department of Physics, National Institute of Technology Rourkela, Rourkela 769008, India
}

(Received 3 November 2020; accepted 30 November 2020; published 18 December 2020)

\begin{abstract}
We study holographic aspects of mixed state entanglement measures in various large $N$ top-down as well as bottom-up confining models. For the top-down models, we consider wrapped $D 3$ and $D 4$ brane gravity solutions, whereas, for the bottom-up confining model, the Einstein-Maxwell-dilaton gravity solution is considered. We study entanglement entropy, mutual information, entanglement wedge cross section, and entanglement negativity for the strip subsystems and find model-independent features of these entanglement measures in all confining theories. The entanglement negativity and entropy exhibit a phase transition at the same critical strip length $L_{\text {crit }}$, at which the order of these measures changes from $\mathcal{O}\left(N^{2}\right)$ to $\mathcal{O}\left(N^{0}\right)$. The entanglement wedge cross section similarly shows an order change at $L_{\text {crit }}$ and exhibits a discontinuous behavior each time a phase transition between different entangling surfaces occurs. We further test the inequality involving mutual information and entanglement wedge cross section and find that the latter always exceeds half of the former.
\end{abstract}

DOI: $10.1103 /$ PhysRevD.102.126022

\section{INTRODUCTION}

Quantum entanglement has recently emerged as an interesting and powerful tool to investigate diverse aspects in theoretical physics, extending from condensed matter to quantum gravity. One of the most commonly used entanglement measures is entanglement entropy. In the past few decades, entanglement entropy has been extensively studied, for example, in condensed matter physics to characterize different quantum phases [1,2], in black hole physics to better understand the Bekenstein-Hawking entropy [3,4], in quantum communications $[5,6]$, etc. Perhaps the most striking development in the discussion of entanglement entropy appeared from the advent of the gauge-gravity duality, in particular, from the Ryu-Takayanagi (RT) proposal for entanglement entropy $[7,8]$.

The RT proposal of entanglement entropy is fundamental to providing an intriguing and deep connection between spacetime geometry, quantum field theories, and quantum information notions. This proposal geometrizes the concept of entanglement entropy and relates the entanglement entropy of the boundary theory to a minimal area of certain bulk codimension-two surface, whose boundary is

\footnotetext{
paruljain@iitb.ac.in

mahapatrasub@nitrkl.ac.in
}

Published by the American Physical Society under the terms of the Creative Commons Attribution 4.0 International license. Further distribution of this work must maintain attribution to the author(s) and the published article's title, journal citation, and DOI. Funded by SCOAP ${ }^{3}$. homologous to the boundary of the subsystem. This proposal has been applied in a variety of systems to probe various physics, such as confinement and deconfinement transitions [9,10], large- $N$ phase transitions [11-13], quench dynamics [14-16], quantum gravity $[17,18]$, holographic quantum error-correcting codes, and tensor networks $[19,20]$, etc., with recent proof of the proposal also appearing in Refs. [21,22]. It is fair to say that the entanglement entropy proposal is one of the most significant and useful suggestions that has emerged from the gauge-gravity duality. It not only provides a deep connection between geometry and quantum information but also provides an elegant way to compute and understand other information theoretic quantities, such as the mutual information, entanglement negativity, entanglement of purifications, etc.

Entanglement entropy, however, unlike for the pure state, is not a good measure of entanglement for the mixed and multipartite states, as it mixes both classical and quantum correlations. For mixed states, new entanglement measures, such as (logarithmic) entanglement negativity, entanglement of purification, entanglement of formation, etc., have been proposed [23-28]. Unfortunately, computation of these measures in quantum field and many-body systems, which generally have a large Hilbert space, is notoriously difficult. From the holographic perspective, there have been a few proposals for these measures. For example, the entanglement of purification has been suggested to be dual to the area of the minimal cross section on the entanglement wedge $[29,30]$, whereas there have been two different proposals for the entanglement negativity. In the first 
proposal, the logarithmic negativity is suggested to be given by the area of an extremal cosmic brane that terminates on the boundary of the entanglement wedge [31,32], and, in the second proposal, it is suggested to be given by certain combinations of the areas of codimension-two minimal bulk surfaces [33-42].

It is important to emphasize that the entanglement wedge cross section appears in the holographic proposals of many information theoretic quantities. Apart from the above mentioned entanglement of purification proposal as well as in the first proposal of the entanglement negativity, recently, it has also appeared in the holographic proposal of the reflected entropy (the entanglement entropy associated to a canonical purification) [43]. Furthermore, these different proposals for the entanglement wedge, in particular, as the holographic dual of the entanglement of purification and reflected entropy, have their own merits and demerits and are in tension with each other [44]. Therefore, it appears that more caution is required while associating an information theoretic notion to the entanglement wedge cross section, and more work is needed to correctly establish the same. In this work, we will not dwell and try to resolve the interpretational issues of the entanglement wedge cross section. Instead, we will compute it in a variety of holographic confining backgrounds and try to investigate whether, like the entanglement entropy, it can also provide signatures and universal results for the (de)confinement.

Because of severe technical difficulties, present both at the analytical as well as at the numerical level, it is generally very hard to obtain any reliable nonperturbative estimate of the entanglement measures in interacting quantum field theories. For these reasons, the analysis of entanglement measures in quantum chromodynamics (QCD)-like theories is rather limited. With the exception of a few lattice related papers [45-48], most of the discussions are based on the holographic proposals, and that too is limited to the entanglement entropy. In Refs. [9,10], the holographic entanglement entropy was computed in the top-down confining models of the gauge-gravity duality, and a phase transition from connected to disconnected entangling surface was found as the size of the entangling region varied. This phase transition, since it causes a change in the order of entanglement entropy [from $\mathcal{O}\left(N^{2}\right)$ to $\mathcal{O}\left(N^{0}\right)$ or vice versa], was suggested as reminiscent of (de)confinement. Importantly, the phase transition and nonanalyticity in the structure of entanglement entropy have also been observed in lattice $S U(N)$ gauge theories [45-48]; see also [49]. The idea of Refs. [9,10] was then applied to many different top-down as well as bottomup confining systems, and results similar to those were obtained [50-68].

Interactions in quantum field theories (QFTs) via the entanglement in quantum states cause quantum information to be dispersed nonlocally across space. To understand better this nonlocal quantum spreading in QCD-like theories, it is not only important to investigate how this structure of shared information changes with the size of the subsystem, but is also necessary to examine the entire structure of the entanglement spectrum, including its mixed and multipartite state measures. However, the discussion of mixed state entanglement measures in confining theories is relativity new. A partial discussion appeared in Ref. [69], where the entanglement wedge cross section in a potentially limited top-down confining model was discussed, whereas no such investigation has been done for the entanglement negativity. In this work, we would like to do a comprehensive analysis of mixed state entanglement measure, including both entanglement wedge cross section and negativity, in a variety of top-down as well as bottomup confining QCD models. The top-down QCD models, although they usually face several limitations in mimicking real QCD and contain undesirable features such as conformal symmetries, the nonrunning coupling constant, additional Hilbert space sector, etc., however, have welldefined gauge-gravity foundations. Whereas the phenomenological bottom-up QCD models, although they lack solid gauge-gravity duality foundations and are generally formatted in an ad hoc way to reproduce desirable features for the boundary QCD, however, can overcome most of the difficulties present in top-down models. By thoroughly investigating pure and mixed state entanglement measures in both top-down as well as bottom-up models, it might not only be possible to obtain universal features of the entanglement in confining theories, but one might also get new predictions from holography, which can be tested via lattice calculations.

In this work, we consider two top-down and one bottomup confining models. The top-down models are obtained by compactifying $D 4$ and $D 3$ branes on a circle [70], whereas for the bottom-up model, we considered the EinsteinMaxwell-dilaton holographic QCD model constructed in Refs. [51,71]. In all cases, the entanglement entropy with one strip goes through a phase transition from a connected to a disconnected surface at the critical strip length $L_{\text {crit }}$. With two equal size disjoint strips, depending on their length $L$ and separation $X$, four different types of minimal area surfaces $\left\{S_{1}, S_{2}, S_{3}, S_{4}\right\}$ appear, which lead to an interesting phase diagram. The mutual information is nonzero only in $S_{2}$ and $S_{3}$ phases and is always a monotonic function of $L$ and $X$. We then study the entanglement wedge cross section and find that it is again nonzero only in $S_{2}$ and $S_{3}$ phases. However, unlike the mutual information, the entanglement wedge cross section not only vanishes discontinuously for large values of $X$ and $L$ but also exhibits a nonanalytic behavior every time a phase transition between different entangling surfaces takes place. A further comparison reveals that the entanglement wedge cross section always exceeds half of the mutual information; i.e., the holographically suggested inequality [29] is satisfied in all confining theories. 
We further investigate the entanglement negativity in confining theories with one and two disjoint intervals. For this purpose, we use the second holographic entanglement negativity proposal [33-42]. The reason for choosing the second proposal is threefold: (i) the first entanglement negativity proposal is closely related to the entanglement wedge cross section, which we will anyhow compute; (ii) it is also computationally easier to implement, as opposed to the first proposal, which requires nontrivial cosmic brane backreaction calculation; and (iii) a direct nontrivial outcome of the second proposal is that it conveys a universal result for the entanglement negativity in all confining theories, which might be possible to test via lattice calculations in the near future. In particular, it suggests that the entanglement negativity is just $3 / 2$ times of the entanglement entropy. This implies that, just like the entanglement entropy, the entanglement negativity also displays a discontinuous behavior at $L_{\text {crit }}$ and undergoes a change in order from $\mathcal{O}\left(N^{2}\right)$ to $\mathcal{O}\left(N^{0}\right)$. Moreover, the proposal also implies an interesting result with two disjoint strips. In particular, unlike the entanglement wedge cross section, it can be nonzero in the $S_{1}$ phase as well.

The paper layout is as follows. In Sec. II, we review various holographic notions of pure and mixed state entanglement measures. In Sec. III, we present the calculations for entanglement entropy, mutual information, entanglement wedge cross section, and entanglement negativity for a top-down confining model obtain by compacting $D 4$ branes on a circle. We repeat the computations of Sec. III in another top-down confining model, this time by compacting $D 3$ branes on a circle, in Sec. IV. In Sec. V, we further discuss these entanglement measures in a phenomenological bottom-up Einstein-Maxwell-dilaton confining model. We end the paper with a discussion and conclusion in Sec. VI.

\section{ENTANGLEMENT MEASURES}

Our main aim in this section is to briefly elucidate the mathematical procedure that is used to calculate various entanglement measures in holographic settings. The list includes entanglement entropy, mutual information, entanglement wedge cross section, and entanglement negativity.

\section{A. Entanglement entropy: One strip}

Entanglement entropy, which is given by the von Neumann entropy

$$
S_{A}=-\operatorname{Tr}_{A} \rho_{A} \ln \rho_{A},
$$

provides the measure of entanglement between pure states. Generally, one uses the nontrivial replica trick to calculate it in quantum field theories [72]. However, implementing the replica technique in nontrivial field theories, such as those containing interactions, is much more tedious.
The holographic idea, on the other hand, provides another pathway to calculate the entanglement entropy in field theories [7,8]. For the case of $\mathrm{AdS}_{d+1} / \mathrm{CFT}_{d}$, the entanglement entropy $S_{A}$ of the subsystem $A$ in $\mathrm{CFT}_{d}$ is given by the Ryu-Takayanagi prescription $[7,8]$

$$
S_{A}=\frac{\mathcal{A}\left(\Gamma_{A}^{\min }\right)}{4 G_{N}^{(d+1)}},
$$

where $\mathcal{A}\left(\Gamma_{A}^{\min }\right)$ is the area of $(d-1)$-dimensional static minimal surface $\left(\Gamma_{A}\right)$ in the bulk whose boundary is homologous to the boundary $\partial A$ of the subsystem $A$. $G_{N}^{(d+1)}$ is the $(d+1)$-dimensional Newton constant. Equivalently, we can also recast the above holographic entanglement entropy formula in the following way:

$$
S_{A}=\frac{1}{4 G_{N}^{(d+1)}} \int_{\Gamma} d^{d-1} \sigma \sqrt{G_{\text {ind }}^{d-1}},
$$

where $G_{\text {ind }}^{d-1}$ is the induced metric on the surface $\Gamma$, which further needs to be appropriately minimized $[9,10]$. Since in this work we are interested in computing the entanglement entropy and other information related quantities in topdown holographic confining theories, where the dual bulk spacetime metric is usually written down in string frame, it is also useful to write down the entanglement entropy expression in string frame metric $[9,10]$ :

$$
S_{A}=\frac{1}{4 G_{N}^{(d+1)}} \int_{\Gamma} d^{d-1} \sigma e^{-2 \phi} \sqrt{G_{\text {ind }}^{d-1}},
$$

where the dilaton field $\phi$ arises because of the frame change.

\section{B. Mutual information: Two strips}

Since the entanglement structure of one interval subsystem is known to display interesting behavior in the confined phases, it is also natural to ask and investigate the entanglement structure with many disjoint intervals. One such natural entanglement measure that appears with two disjoint intervals is the mutual information [73-75]. For two subsystems $\left(A_{1}\right.$ and $\left.A_{2}\right)$, the mutual information is defined as the amount of information that $A_{1}$ and $A_{2}$ can share and is defined in terms of entanglement entropy as

$$
I\left(A_{1}, A_{2}\right)=S_{A_{1}}+S_{A_{2}}-S_{A_{1} \cup A_{2}},
$$

where $S_{A_{1}}, S_{A_{2}}$, and $S_{A_{1} \cup S_{A_{2}}}$ are as usual the entanglement entropies of subsystems $A_{1}, A_{2}$, and their union, respectively. It is quite obvious from Eq. (2.5) that the mutual information is zero for two uncorrelated systems. Importantly, the mutual information does not suffer from the ambiguities associated with the entanglement entropy and can provide more information than the entanglement 
entropy alone. In particular, mutual information is UV finite and does not contain the usual UV divergences. Moreover, the subadditivity property of the entanglement entropy also ensures that the mutual information is nonnegative; i.e., $I\left(A_{1}, A_{2}\right)$ provides an upper bound on the correlation functions between operators in $A_{1}$ and $A_{2}$. For more discussion on the mutual information, see Refs. [76-83]. For the discussion on mutual information and two-strip entanglement phase diagram in top-down and bottom-up confining models, see Refs. [52,55]. Similarly, we can also define other entanglement measures, such as $n$-partite information, with more disjoint intervals

$$
\begin{aligned}
I^{n}\left(A_{\{i\}}\right)= & \sum_{i=1}^{n} S_{A_{i}}-\sum_{i<j}^{n} S_{A_{i} \cup A_{j}}+\sum_{i<j<k}^{n} S_{A_{i} \cup A_{j} \cup A_{k}}-\cdots \\
& -(-1)^{n} S_{A_{1} \cup A_{2} \cup \cdots \cup A_{n}} .
\end{aligned}
$$

In this work, we will concentrate only on mutual information, as the results for the $n$-partite information can be analogously obtained.

\section{Entanglement wedge cross section}

Entanglement entropy serves as a good measure for the entanglement in the case of pure states, but it fails in the case of mixed states. Therefore, it would be an interesting question to investigate how the mixed state entanglement measures behave in confining theories. One such mixed state entanglement measure is the entanglement of purification, which in the holographic context is suggested to be given by the minimal area of the entanglement wedge cross section $[29,30] .{ }^{1}$ However, as mentioned in the introduction, there are other holographic interpretations of the entanglement wedge as well (such as the holographic dual of the reflected entropy), and these different interpretations, unfortunately, do not exactly correlate with each other. In this work, we will mainly concentrate on the entanglement wedge cross section, without worrying too much about its interpretational issues.

To compute the entanglement wedge cross section holographically, we follow the prescription suggested in Refs. [29,30]. We first consider two subsystems $A$ and $B$ with no overlap on the $d$-dimensional boundary. The RyuTakayanagi minimal surfaces for $A$ and $B$ and their union $A B=A \cup B$ are denoted by $\Gamma_{A}^{\min }, \Gamma_{B}^{\min }$, and $\Gamma_{A B}^{\min }$, respectively. The $d$-dimensional entanglement wedge $M_{A B}$ in the $(d+1)$-dimensional bulk is then described as a region which is bounded by $A, B$, and $\Gamma_{A B}^{\min }$; i.e., the entanglement wedge $M_{A B}$ is the bulk region whose boundary is

$$
\partial M_{A B}=A \cup B \cup \Gamma_{A B}^{\min } .
$$

\footnotetext{
${ }^{1}$ For some related discussion on the entanglement wedge cross section and entanglement of purification, see Refs. [84-97].
}

One should note that the entanglement wedge is a bulk codimension-zero region. However, here it turns out to be $d$ dimensional as we are considering static bulk configuration. Also note that if $A$ and $B$ are very small or have large separation between them, then $M_{A B}$ will have a disconnected form.

We next divide $\Gamma_{A B}^{\min }$ as follows:

$$
\Gamma_{A B}^{\min }=\Gamma_{A B}^{(A)} \cup \Gamma_{A B}^{(B)},
$$

and we further define

$$
\begin{gathered}
\tilde{\Gamma}_{A}=A \cup \Gamma_{A B}^{(A)}, \\
\tilde{\Gamma}_{B}=A \cup \Gamma_{A B}^{(B)} .
\end{gathered}
$$

Note that, as a result of Eqs. (2.8) and (2.9), the boundary of the entanglement wedge $M_{A B}$ is now divided into two parts:

$$
\partial M_{A B}=\tilde{\Gamma}_{A} \cup \tilde{\Gamma}_{B}
$$

A pictorial representation of the holographic entanglement wedge is shown in Fig. 1. Next, look for the minimum surface $\Sigma_{A B}^{\min }$ subjected to conditions

(i) $\partial \Sigma_{A B}^{\min }=\partial \tilde{\Gamma}_{A}=\partial \tilde{\Gamma}_{B}$,

(ii) $\Sigma_{A B}^{\min }$ is homologous to $\tilde{\Gamma}_{A}$ inside $M_{A B}$.

The holographic entanglement wedge cross section $E_{W}\left(\rho_{A B}\right)$ is then simply defined as the area of $\Sigma_{A B}^{\min }$ divided by $4 G_{N}^{d+1}$ :

$$
E_{W}\left(\rho_{A B}\right)=\min _{\Gamma_{A B}^{(A)} \subset \Gamma_{A B}^{\min }}\left[\frac{\mathcal{A}\left(\Sigma_{A B}^{\min }\right)}{4 G_{N}^{(d+1)}}\right] .
$$

Note that for given $A$ and $B$ subsystems, as we will also see later in this work, there can be many possible $\Sigma_{A B}^{\min }$ surfaces. In those cases, the entanglement wedge cross section is given by the surface that has the minimum area.

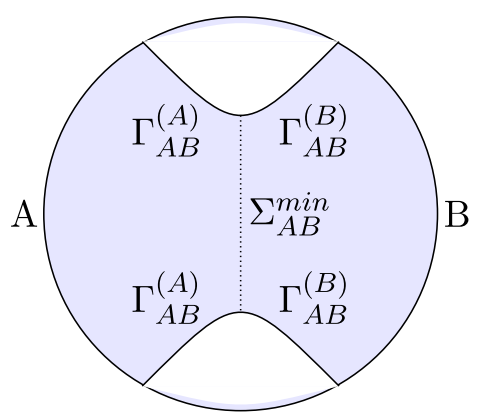

FIG. 1. The region in blue is the entanglement wedge $M_{A B}$ corresponding to a pure state. The dotted surface is $\Sigma_{A B}$ which divides $M_{A B}$ into two parts. 
To summarize, $E_{W}\left(\rho_{A B}\right)$ is the minimal surface area of the entanglement wedge $M_{A B}$ connecting $A$ and $B$.

\section{Entanglement negativity}

In the previous section, we talked about the entanglement wedge cross section (and its holographic definition) as a suitable measure for the mixed state entanglement. Another quantum information quantity which is also known to capture mixed state entanglement is the entanglement negativity. This is defined in the quantum manybody system as $[23,24]$

$$
\mathcal{N}=\frac{\left\|\rho^{T_{2}}\right\|-1}{2},
$$

where $\left\|\rho^{T_{2}}\right\|$ is the trace norm of the partially transposed reduced density matrix $\rho^{T_{2}}$ and this trace norm is generally given by the sum of the absolute eigenvalues of $\rho^{T_{2}}$. There also exists a close variant of entanglement negativity, called logarithmic negativity, which is also frequently used in the quantum information community. This is defined as

$$
\mathcal{E}=\ln \left\|\rho^{T_{2}}\right\|=\ln \operatorname{Tr}\left|\rho^{T_{2}}\right| .
$$

The (logarithmic) entanglement negativity has been computed in a variety of quantum many-body and field theory systems and has been widely used in the condensed matter and quantum information community; see, for example, Refs. [98-114]. The primary reason for this is that it provides an upper bound on the distillable entanglement. Holographically, to the best of our knowledge, two different (yet equivalent) proposals for entanglement negativity have been suggested. In the first proposal, the logarithmic negativity is suggested to be given by the area of an extremal cosmic brane that terminates on the boundary of the entanglement wedge [31,32]. In the second proposal, the logarithmic negativity is suggested to be given by certain combinations of the areas of codimension-two minimal bulk surfaces [33-42]. Both these proposals have been tested in diverse physical situations and have shown to reproduce exact known results for the negativity in CFTs. Since the first proposal is very closely related to the entanglement wedge cross section, which we will anyhow be going to investigate, it might be more informative, and at the time complementary as well, if the second holographic proposal is adopted for the computation of entanglement negativity. It is also computationally slightly more straightforward to implement the second proposal, as opposed to the first proposal, which requires nontrivial cosmic brane backreaction calculation. Moreover, as we will see later on, the second proposal also advocates a universal result for the entanglement negativity in all holographic confining theories, which might be possible to check via lattice calculations. For these reasons, we will take the second proposal for the entanglement negativity in this paper. It would certainly be interesting to explicitly compute the entanglement negativity in confining theories using the first proposal and find its similarities and differences with the second proposal. We leave this interesting exercise for future work.

To compute the holographic logarithmic negativity for a single interval using Refs. [33-35], first, a bipartition of the system into $A$ and its complement $A^{c}$ is considered in $\mathrm{CFT}_{d}$. Next, two finite length intervals $B_{1}$ and $B_{2}$ are taken adjacent on both sides of $A$ such that $B=B_{1} \cup B_{2}$. Refer to Fig. 2. If the corresponding codimension-two bulk static minimal surfaces in $\mathrm{AdS}_{d+1}$ are denoted by $\Gamma_{A}, \Gamma_{B_{1}}$, and $\Gamma_{B_{2}}$, then the holographic entanglement negativity for the bipartite system $\left(A \cup A_{c}\right)$ is suggested to be given by the following combination of the areas of the minimal bulk surfaces:

$$
\begin{aligned}
\mathcal{E}= & \lim _{B \rightarrow A^{c}} \frac{3}{16 G_{N}^{(d+1)}}\left[2 \mathcal{A}\left(\Gamma_{A}\right)+\mathcal{A}\left(\Gamma_{B_{1}}\right)+\mathcal{A}\left(\Gamma_{B_{2}}\right)-\mathcal{A}\left(\Gamma_{A \cup B_{1}}\right)\right. \\
& \left.-\mathcal{A}\left(\Gamma_{A \cup B_{2}}\right)\right]
\end{aligned}
$$

where in the above equation it should be understood that both $B_{1}$ and $B_{2}$ are extending to infinity such that $B=B_{1} \cup B_{2}=A^{c}$. In the $\mathrm{AdS}_{3} / \mathrm{CFT}_{2}$ context, the above formula was deduced from how the four-point twist correlation functions factorize in the large central charge limit. In particular, the entanglement negativity in $\mathrm{CFT}_{2}$ is given by a specific four-point twist correlator in the $n$-sheeted Riemann surface [98,99]. This four-point correlation function factorizes into various combinations of two-point functions in the large central charge limit, which can then be mapped, using the AdS/CFT dictionary, to the

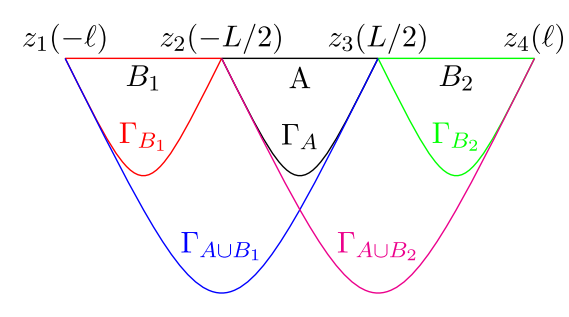

Single interval configuration

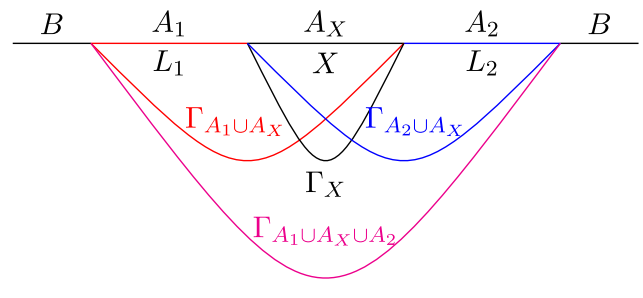

Two disjoint intervals configuration

FIG. 2. Pictorial representation of various minimal area surfaces that contribute in the entanglement negativity. 
length of the geodesic anchored on the boundary points and extending into the bulk. In this way, the authors of Refs. $[33,34]$ arrived at the above formula for the entanglement negativity in $\mathrm{CFT}_{2}$ (and its $d$-dimensional extension). This entanglement negativity formula, though an unproven conjectured proposal, in contrast with the RyuTakayanagi entanglement entropy proposal, however, does reproduce mixed and pure state results of the entanglement negativity in CFT. For more details on this entanglement negativity conjecture, see Refs. [33,34].

One can also write the above formula in terms of the entanglement entropy using the Ryu-Takayanagi formula [Eq. (2.2)]:

$$
\mathcal{E}=\lim _{B \rightarrow A^{c}} \frac{3}{4}\left[2 S_{A}+S_{B_{1}}+S_{B_{2}}-S_{A \cup B_{1}}-S_{A \cup B_{2}}\right] .
$$

In the subsequent sections, we will take the above formula to compute the entanglement negativity in a variety of top-down as well as bottom-up confining backgrounds. As we will see, the above entanglement negativity formula reduces to a simpler expression and suggests a universal relation in all confining theories.

For two disjoint intervals $A_{1}$ and $A_{2}$, the negativity is similarly suggested to be given by the following combination of the minimal area surfaces:

$$
\mathcal{E}=\frac{3}{4}\left[S_{A_{1} \cup A_{X}}+S_{A_{X} \cup A_{2}}-S_{A_{1} \cup A_{2} \cup A_{X}}-S_{A_{X}}\right],
$$

where $X$ is the separation between the two intervals. Refer to Fig. 2 for more details on the surfaces appearing in Eq. (2.17). Again, this is an unproven conjectured formula which is obtained by analyzing the factorization of a fourpoint twist correlation function in terms of two-point correlations in the large central charge limit [39,41]. This conjectured formula, however, again reproduces the desirable entanglement negativity results of the boundary CFT.

\section{D4 BRANES ON A CIRCLE}

The first top-down holographic confining model we consider is obtained by putting $D 4$ branes on a circle. As is well known, the low-energy dynamics of $N_{c}$ coincidental $D 4$ branes in type IIA string theory is given by $(4+1)$-dimensional $U\left(N_{c}\right)$ supersymmetric Yang-Mills theory with 't Hooft coupling $\lambda=g_{s} N_{c} l_{s}$. This theory can be reduced to $(3+1)$ dimensions with broken supersymmetry by compacting one of the directions along the brane, say, $x^{4}$, on a circle with radius $R_{4}\left(x^{4} \sim x^{4}+2 \pi R_{4}\right)$. The low-energy dynamics of the reduced system is then given by the dimensionless parameter $\lambda_{4}=\lambda / R_{4}$ [70]. The condition $\lambda_{4} \gg 1$ allows us to investigate this system using its dual gravitational picture. The near-horizon geometry of the $D 4$ branes is [70]

$$
\begin{aligned}
d s^{2}= & \left(\frac{U}{R}\right)^{3 / 2}\left[\left(\frac{R}{U}\right)^{3} \frac{d U^{2}}{f(U)}+d x^{\mu} d x_{\mu}\right]+R^{3 / 2} U^{1 / 2} d \Omega_{4}^{2} \\
& +\left(\frac{U}{R}\right)^{3 / 2} f(U)\left(d x^{4}\right)^{2}, \\
e^{-2 \phi}= & \left(\frac{R}{U}\right)^{3 / 2}, \quad f(U)=1-\left(\frac{U_{0}}{U}\right)^{3}, \\
U_{0}= & \frac{4 \pi \lambda}{9 R_{4}^{2}}, \quad R^{3}=\pi \lambda .
\end{aligned}
$$

Here $R$ is the AdS length scale. Note that this geometry forms a cigar shape in $\left(U, x^{4}\right)$ coordinates, with the radius of the $x^{4}$ circle going to zero as $U \rightarrow U_{0}$. The radial value $U_{0}$ therefore introduces a mass gap in the theory.

\section{A. Entanglement entropy: One strip}

The entanglement entropy has already been computed for this system in Ref. [9]. Here, we will reproduce their results to set the stage for the later sections. To calculate the entanglement entropy, we consider a strip subsystem of length $L$ and define the subsystem domain as $-L / 2 \leq$ $x_{1}=x \leq L / 2,0 \leq x_{2} \leq L_{2}$, and $0 \leq x_{3} \leq L_{3}$. The parametrization $U=U(x)$ leads to the following expression for the entanglement entropy (2.4):

$S_{A}=\frac{L_{2} L_{3} \omega_{4}\left(2 \pi R_{4}\right)}{4 G_{N}^{(10)}} \int d x R^{3 / 2} U^{5 / 2} \sqrt{f(U)+\left(\frac{R}{U}\right)^{3} U^{\prime 2}}$,

where $\omega_{4}$ is the area of unit four sphere. It turns out that there are actually two surfaces which minimize the above entanglement action: a ( $U$-shaped) connected and a disconnected surface. The expression of the entanglement entropy of the connected surface is simply given by

$S_{A}^{\mathrm{con}}=\frac{L_{2} L_{3}}{2 G_{N}^{(10)}} \frac{32 \pi^{3} R^{9 / 2}}{9} \int_{U_{*}}^{U_{\infty}} d U \frac{U^{7 / 2}}{\sqrt{U_{0}}} \frac{\sqrt{f(U)}}{\sqrt{U^{5} f(U)-U_{*}^{5} f\left(U_{*}\right)}}$,

where $U_{*}$ is the turning point of the connected surface at which $\left.U^{\prime}(x)\right|_{U=U_{*}}=0$ and is related to the strip length $L$ in the following way:

$$
\begin{aligned}
L\left(U_{*}\right)= & 2 R^{3 / 2} \int_{U_{*}}^{U_{\infty}} d U \frac{U_{*}^{5 / 2}}{U^{3 / 2}} \sqrt{\frac{f\left(U_{*}\right)}{f(U)}} \\
& \times \frac{1}{\sqrt{U^{5} f(U)-U_{*}^{5} f\left(U_{*}\right)}} .
\end{aligned}
$$

Similarly, the entanglement entropy for the disconnected surface is given by 


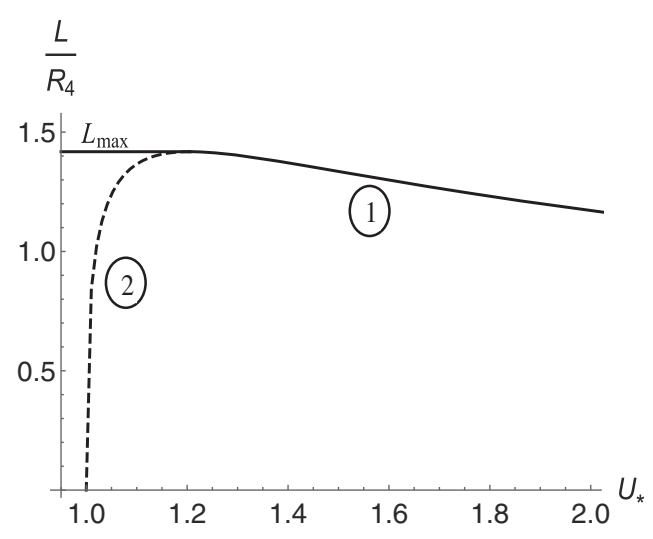

FIG. 3. The behavior of strip length $L$ as a function of $U_{*}$.

$$
S_{A}^{\text {discon }}=\frac{L_{2} L_{3}}{2 G_{N}^{(10)}} \frac{32 \pi^{3} R^{9 / 2}}{9} \int_{U_{0}}^{U_{\infty}} d U \frac{U}{\sqrt{U_{0}}} .
$$

Note that the disconnected entanglement entropy does not depend on the strip length. This result will have profound implications in the entanglement structure of the confined phase.

We now present the numerical results for the entanglement entropy. For the numerical purpose, we take $U_{0}=1$. The results are shown in Figs. 3 and 4, where the variation of $L$ with respect to $U_{*}$ and connected and disconnected entanglement entropy difference $\left(\triangle S_{A}=S_{A}^{\text {con }}-S_{A}^{\text {discon }}\right)$ with respect to $L$, respectively, are plotted. ${ }^{2}$ Note that, at a given $L$, there exist three minimal area surfaces: one disconnected and two connected. The first connected surface (1) (shown by a solid line) is nearer to the boundary compared to the second connected surface (2) (shown by a dashed line). These connected surfaces exist only below a maximum length $L_{\max } \simeq 1.418 R_{4}$, and above $L_{\max }$ they cease to exist. In particular, there is no solution for the connected surface above $L_{\max }$, and only the disconnected surface remains. The second connected surface (dashed line) actually corresponds to a saddle point, and its area (and, hence, the entanglement entropy) is always higher than the first connected surface (solid line).

Importantly, a change in sign in $\triangle S_{A}$ appears as the strip length $L$ varies. In particular, $\triangle S_{A}$ is negative for small $L$, indicating that the connected surface (3) has the lowest entanglement entropy for the small subsystem, whereas $\triangle S_{A}$ is positive for large $L$, indicating that the disconnected surface has the lowest entanglement entropy for the large subsystem. Therefore, a phase transition takes place between connected and disconnected entanglement entropies as $L$ increases. This phase transition occurs at $L=L_{\text {crit }} \simeq 1.288 R_{4}$. Since the entanglement entropy of the disconnected surface is independent of $L$, it gives us the following important result:

\footnotetext{
${ }^{2}$ Here, and in the subsequent subsections, we have set $L_{2} L_{3} / 4 G_{N}^{(10)}=1$.
}

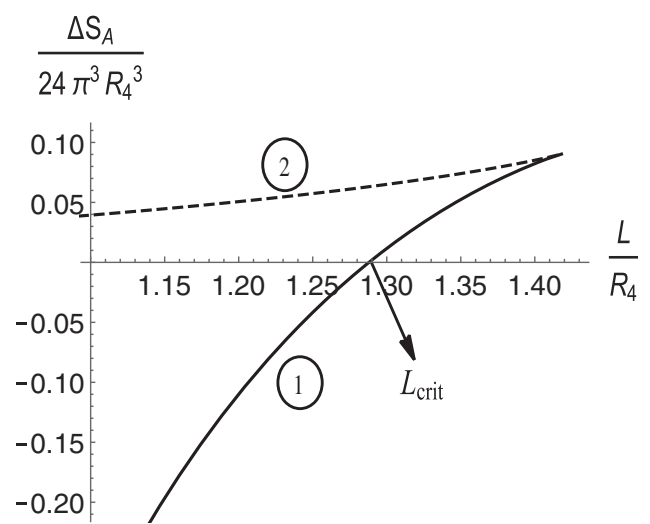

FIG. 4. $\triangle S_{A}=S_{A}^{\text {con }}-S_{A}^{\text {discon }}$ as a function of strip length $L$.

$$
\begin{aligned}
\frac{\partial S_{A}}{\partial L} & \propto \frac{1}{G_{N}^{(10)}}=\mathcal{O}\left(N^{2}\right) \quad \text { for } L<L_{\text {crit }} \\
& \propto \frac{1}{\left[G_{N}^{(10)}\right]^{0}}=\mathcal{O}\left(N^{0}\right) \quad \text { for } L>L_{\text {crit }} .
\end{aligned}
$$

The above type of connected-disconnected phase transition, where the order of the entanglement entropy changes from $\mathcal{O}\left(N^{2}\right)$ at small $L$ to $\mathcal{O}\left(N^{0}\right)$ at large $L$, was suggested to be reminiscent of the confinement-deconfinement transition in QCD [9]. Moreover, the fact that the order of colored gluon degrees of freedom $\left[\mathcal{O}\left(N^{2}\right)\right]$ above the deconfinement temperature and color neutral confined degrees of freedom $\left[\mathcal{O}\left(N^{0}\right)\right]$ below the deconfinement temperature match well with the connected and disconnected entanglement entropies further naturally led to the interpretation of the critical subsystem size as the inverse deconfinement temperature $\left(T_{D}\right)$, i.e., $T_{D} \propto 1 / L_{\text {crit }}$. In Ref. [9], the value of $L_{\text {crit }}$ was computed for different confining models, and the relation $L_{\text {crit }}=\mathcal{O}\left(\Lambda_{\mathrm{IR}}^{-1}\right)$ was found. This further suggested that the entanglement entropy can act as a probe to diagnose confinement. ${ }^{3}$ This work was then generalized to many other top-down as well as bottom-up holographic QCD models, and similar results were found in all the cases [50-68].

\section{B. Mutual information: Two strips}

Having discussed the entanglement structure with one strip, we now move on to discuss it with two strips. Here, for simplicity, we concentrate only on equal size strips $\left(L_{1}=L_{2}=L\right)$. The entanglement structure with two strips is much more interesting than with one strip. In particular, depending upon the strip length $L$ and the distance between them $X$, there are now four different entangling surfaces which compete with each other. These surfaces are shown in Fig. 5. With two strips, both connected $\left(S_{1}\right.$ and $\left.S_{2}\right)$ and

\footnotetext{
${ }^{3}$ It has been suggested recently that the entanglement entropy signals only the presence of a mass gap rather than the confinement [115].
} 


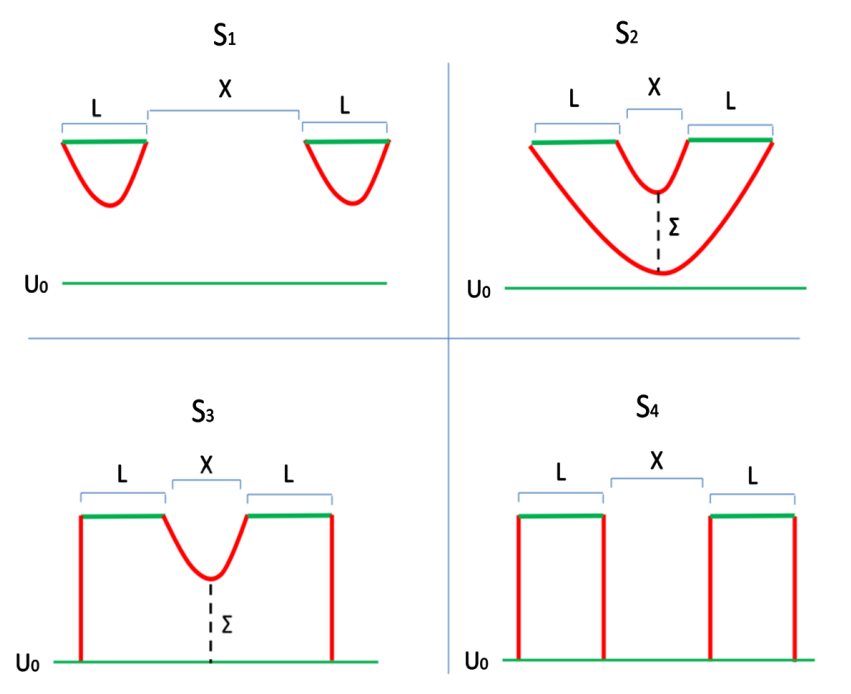

FIG. 5. Sketch of four different minimal area surfaces that can occur for the cases of two parallel strips of equal length $L$ separated by a distance $X$ in the confining background. The black dashed lines denote a nonzero connected entanglement wedge.

disconnected $\left(S_{4}\right)$ as well as a combination of connected and disconnected $\left(S_{3}\right)$ surfaces can appear.

The entanglement entropy expressions of these surfaces are given as

$$
\begin{aligned}
& S_{1}(L, X)=2 S_{A}^{\mathrm{con}}(L), \\
& S_{2}(L, X)=S_{A}^{\mathrm{con}}(X)+S_{A}^{\mathrm{con}}(2 L+X), \\
& S_{3}(L, X)=S_{A}^{\mathrm{con}}(X)+S_{A}^{\text {discon }}, \quad S_{4}(L, X)=2 S_{A}^{\text {discon }},
\end{aligned}
$$

where $S_{A}^{\text {con }}$ and $S_{A}^{\text {discon }}$ are as usual the entanglement entropies of the connected and disconnected surfaces, respectively, with one strip.

Depending on the magnitude of $L$ and $X$, there appear various phase transitions between $S_{1}, S_{2}, S_{3}$, and $S_{4}$. In Fig. 6, the complete phase diagram in the phase space of $(L, X)$ is shown. For small $X, L \ll L_{\text {crit }}$, it is usually the connected $S_{1}$ surface which has the lowest area (and entanglement entropy). The area of the connected $S_{2}$ surface, however, becomes smaller as $L$ increases. With a further increase in $L$, keeping $X\left(\ll L_{\text {crit }}\right)$ small, the area of the $S_{3}$ becomes smaller and a phase transition from $S_{2}$ to $S_{3}$ takes place. For $X=0$, this phase transition occurs at $L=L_{\text {crit }} / 2$. For a general value of $X$, the phase transition line between $S_{2}$ and $S_{3}$ is given by $2 L+X=L_{\text {crit, }}$, as is obvious from Eq. (3.7). Finally, the $S_{4}$ configuration becomes more favorable with a further increase in both $X, L>L_{\text {crit }}$.

Interestingly, with two strips, two tricritical points appear where three minimal area entangling surfaces coexist. These are denoted by black dots in Fig. 6. At the first tricritical point $L_{c 1}$, the phases $\left(S_{1}, S_{2}\right.$, and $\left.S_{3}\right)$ coexist,

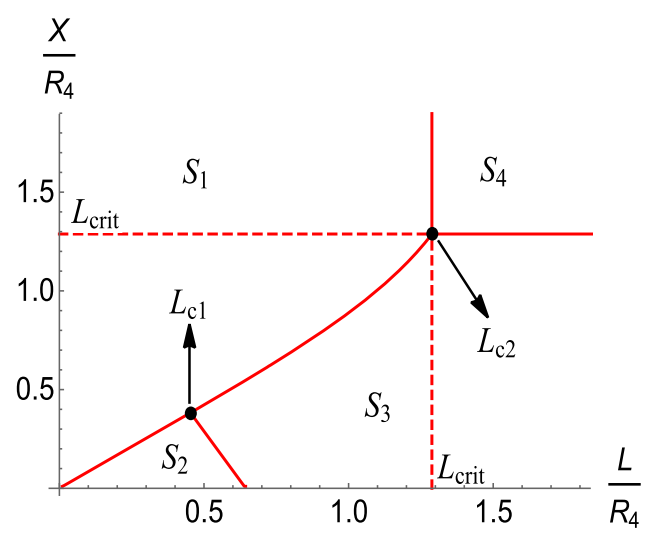

FIG. 6. The entanglement entropy phase diagram of various minimal area surfaces for the case of two strips of equal length $L$ separated by a distance $X$ in the confining background. These four different phases correspond to the four bulk surfaces in Fig. 5.

whereas at the second tricritical point $L_{c 2}$, the phases $\left(S_{1}\right.$, $S_{3}$, and $S_{4}$ ) coexist. The coordinates of these tricritical points are $\left(L / R_{4}=0.454, X / R_{4}=0.381\right)$ and $\left(L / R_{4}=\right.$ $1.288, X / R_{4}=1.288$ ), respectively. Importantly, the tricritical points and two-strip phase diagram again suggest nonanalyticity in the entanglement structure.

Let us now probe the structure of mutual information in the above mentioned four entangling phases. In these phases, the mutual information simply reduces to

$$
\begin{aligned}
I_{1}(L, X)= & S_{A}^{\mathrm{con}}(L)+S_{A}^{\mathrm{con}}(L)-2 S_{A}^{\mathrm{con}}(L)=0, \\
I_{2}(L, X)= & S_{A}^{\mathrm{con}}(L)+S_{A}^{\mathrm{con}}(L)-S_{A}^{\mathrm{con}}(X) \\
& -S_{A}^{\mathrm{con}}(2 L+X) \geq 0 \\
I_{3}(L, X)= & S_{A}^{\mathrm{con}}(L)+S_{A}^{\mathrm{con}}(L)-S_{A}^{\mathrm{con}}(X)-S_{A}^{\mathrm{discon}} \geq 0, \\
I_{4}(L, X)= & S_{A}^{\text {discon }}+S_{A}^{\text {discon }}-2 S_{A}^{\mathrm{discon}}=0 .
\end{aligned}
$$

Here, the range of $X$ and $L$ in $I_{2}$ and $I_{3}$ should be understood to be restricted in their respective phases. The above equations also imply

$$
\begin{array}{lll}
\frac{\partial I_{1}}{\partial L} \propto \frac{1}{G_{N}^{0}}=\mathcal{O}\left(N^{0}\right), & \frac{\partial I_{2}}{\partial L} \propto \frac{1}{G_{N}}=\mathcal{O}\left(N^{2}\right), \\
\frac{\partial I_{3}}{\partial L} \propto \frac{1}{G_{N}}=\mathcal{O}\left(N^{2}\right), & \frac{\partial I_{4}}{\partial L} \propto \frac{1}{G_{N}^{0}}=\mathcal{O}\left(N^{0}\right) .
\end{array}
$$

As expected, the mutual information is zero for the phases containing two largely separated subsystems, i.e., for $S_{1}$ and $S_{4}$ phases, whereas for the phases $S_{2}$ and $S_{3}$ it is nonzero and finite. The variation of mutual information with respect to strip length $L$ and separation length $X$ are shown in Figs. 7 and 8. It turns out that the mutual information is not only a smooth function of $L$ and $X$ but also behaves smoothly as it passes from one phase to another. For instance, as shown in Fig. 7, it connects 


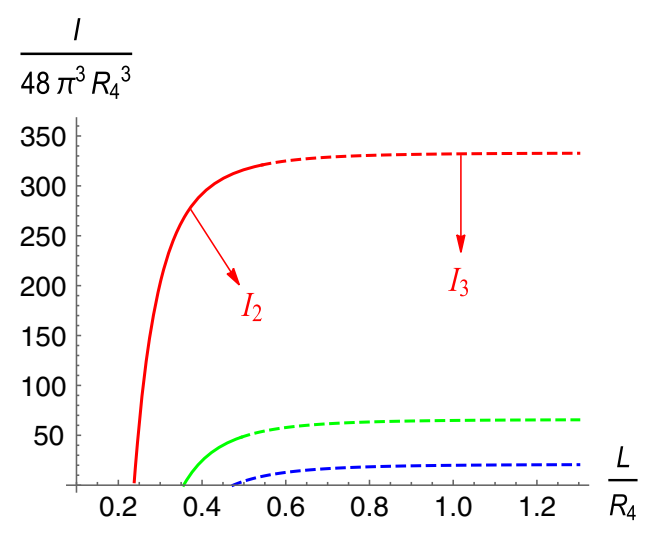

FIG. 7. Mutual information of $S_{2}$ and $S_{3}$ phases as a function of $L$. The solid lines correspond to $I_{2}$, whereas the dashed lines correspond to $I_{3}$. The red, green, and blue lines correspond to separation length $X / R_{4}=0.2,0.3$, and 0.4 , respectively.

smoothly between $S_{2}$ and $S_{3}$ phases and no discontinuity arises as the $S_{2} / S_{3}$ critical line is approached. Similarly, the mutual information also smoothly goes to zero as $S_{1}$ (or $S_{4}$ ) phase is approached from the $S_{2}$ (or $S_{3}$ ) side. This is shown in Fig. 8.

An important point to note is that, unlike the entanglement entropy, the order of the mutual information may or may not change as we go from one phase to another. For instance, a change in the order of mutual information [O $\mathcal{O}\left(N^{2}\right)$ to $\left.\mathcal{O}\left(N^{0}\right)\right]$ occurs as we go from $S_{2}$ to $S_{1}$ phase (by increasing $X)$; however, no such change in the order occurs from $S_{2}$ to $S_{3}$ phase (by increasing $L$ ). It all depends on the particular phases involved in the transition.

\section{Entanglement wedge cross section}

We now move on to discuss the entanglement wedge cross section $E_{W}$ in the current confining background. For this purpose, note that the surface which divides the entanglement wedge into two parts, associated with $A$ and

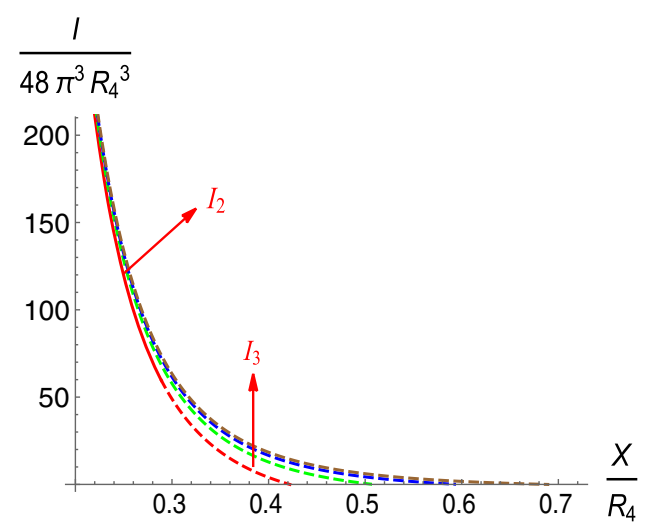

FIG. 8. Mutual information of $S_{2}$ and $S_{3}$ phases as a function of $X$. The solid and dashed lines correspond to $I_{2}$ and $I_{3}$, respectively. The red, green, blue, and brown lines correspond to $L / R_{4}=0.5,0.6,0.7$, and 0.8 , respectively.
$B$ (see Fig. 1), can be identified by symmetry consideration to be a vertical flat surface $\Sigma$. Therefore, the symmetry of the strip configuration ensures that the entanglement wedge cross section in the confining background is given by the area of a constant $x$ hypersurface located in the middle of the strips (see Fig. 5). The induced metric on $\Sigma$ (a surface defined with $t=$ const and $x=$ const) is then given by

$$
\begin{aligned}
\left(d s^{2}\right)_{\Sigma}^{\text {ind }}= & \left(\frac{U}{R}\right)^{3 / 2}\left[\left(\frac{R}{U}\right)^{3} \frac{d U^{2}}{f(U)}+d x^{i} d x_{i}\right] \\
& +R^{3 / 2} U^{1 / 2} d \Omega_{4}^{2}+\left(\frac{U}{R}\right)^{3 / 2} f(U)\left(d x^{4}\right)^{2},
\end{aligned}
$$

where $i=1,2$. Correspondingly, the entanglement wedge cross section for the dual confining theory is given by

$$
E_{W}=\frac{1}{4 G_{N}^{(10)}} \int d^{8} \sigma e^{-2 \phi} \sqrt{g_{\Sigma}^{\text {ind }}}
$$

where we have again used the fact that the induced metric in Eq. (3.10) is in the string frame. As mentioned above, there are, in fact, four different minimal area surfaces for two parallel strips. Since there is no correlation between the two disjointed strips in $S_{1}$ and $S_{4}$ phases, the corresponding entanglement wedge cross section is trivially zero. For the connected $S_{2}$ and $S_{3}$ phases, the entanglement wedge cross section is nonzero. For the $S_{2}$ phase, it is given by

$$
\begin{aligned}
E_{W}^{2} & =\frac{L_{2} L_{3} \omega_{4}\left(2 \pi R_{4}\right)}{4 G_{N}^{(10)}} \int_{U_{*}(2 L+X)}^{U_{*}(X)} d U R^{3} U \\
& =\frac{L_{2} L_{3}}{4 G_{N}^{(10)}} \frac{\left(12 \pi^{3} R_{4}^{3} U_{0}\right)}{2}\left[U_{*}^{2}(X)-U_{*}^{2}(2 L+X)\right] .
\end{aligned}
$$

Similarly, for the $S_{3}$ phase it is given by

$$
\begin{aligned}
E_{W}^{3} & =\frac{L_{2} L_{3} \omega_{4}\left(2 \pi R_{4}\right)}{4 G_{N}^{(10)}} \int_{U_{0}}^{U_{*}(X)} d U R^{3} U \\
& =\frac{L_{2} L_{3}}{4 G_{N}^{(10)}} \frac{\left(12 \pi^{3} R_{4}^{3} U_{0}\right)}{2}\left[U_{*}^{2}(X)-U_{0}^{2}\right] .
\end{aligned}
$$

From the above definition, it is clear that $E_{W}^{2}$ and $E_{W}^{3}$ are both positive as $U_{*}(x) \geq U_{*}(2 L+X) \geq U_{0}$.

In Fig. 9, we have shown the variation of entanglement wedge as a function of $X$ for a few values of $L$. Here, nearcritical values of $L$ are chosen so that $E_{W}$ behavior near the $S_{1} / S_{3}$ and $S_{3} / S_{4}$ phase transition points can be seen. We find that $E_{W}$ is a monotonic function of $X$ and is discontinuous at the transition point. In particular, $E_{W}$ does not go to zero as the $S_{3} / S_{4}$ critical line is approached from the $S_{3}$ side (blue curve). The discontinuous behavior can also be seen mathematically from Eq. (3.13). Notice that, for $E_{W}^{3}$ to be zero at the $S_{3} / S_{4}$ critical line, one requires 


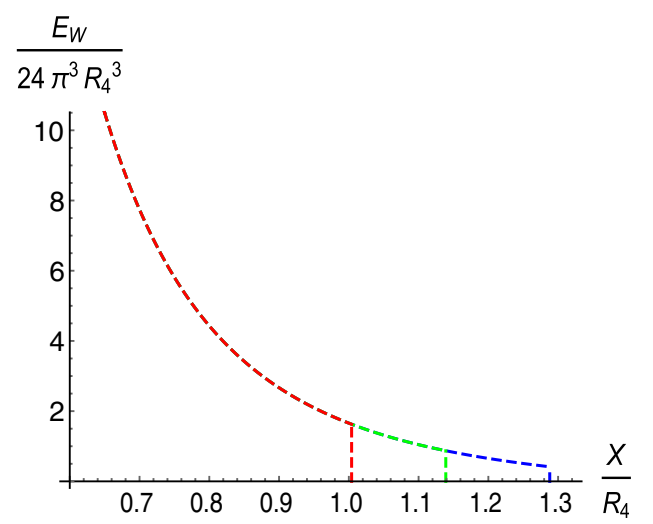

FIG. 9. $\quad E_{W}$ as a function of separation length $X$ for different values of strip length $L$. Here blue, green, and red curves correspond to $L / R_{4}=1.3>L_{\text {crit }}, 1.2$, and 1.1 , respectively.

$U_{*}\left(X=L_{\text {crit }}\right)=U_{0}$. However, $U_{*}\left(L_{\text {crit }}\right)$ is always larger than $U_{0}$ in the current confining background, which implies that $E_{W}^{3}$ is greater than zero at $X_{\text {crit }}$. Similarly, $E_{W}^{3}$ also does not go to zero at the $S_{1} / S_{3}$ transition line (red curve). This analysis suggests that, in the confining phase, the entanglement wedge cross section vanishes discontinuously for large values of $X$ and $L$.

It is also interesting to investigate the behavior of $E_{W}$ near the $S_{2} / S_{3}$ critical line, considering that $E_{W}$ is nonzero in both these phases. The results are shown in Fig. 10. Here, we have considered a particular line, $X=0.7 L$, so that the behavior of $E_{W}$ in $S_{2}, S_{3}$, and $S_{4}$ phases can be probed simultaneously. $E_{W}$ again turns out to be discontinuous at the $S_{2} / S_{3}$ transition line. This can also again be seen mathematically from Eqs. (3.12) and (3.13). In particular, the condition $U_{*}^{2}(2 L+X) \neq U_{0}$ ensures that $E_{W}^{2}$ and $E_{W}^{3}$ do not correspond to the same value at the $S_{2} / S_{3}$ critical point. Moreover, as the $S_{2} / S_{3}$ critical point is approach from the $S_{2}$ side, there is an upward jump in the $E_{W}$ value, i.e., $E_{W}^{3}>E_{W}^{2}$, suggesting that the area of the entanglement wedge grows at the critical point. This result can again be

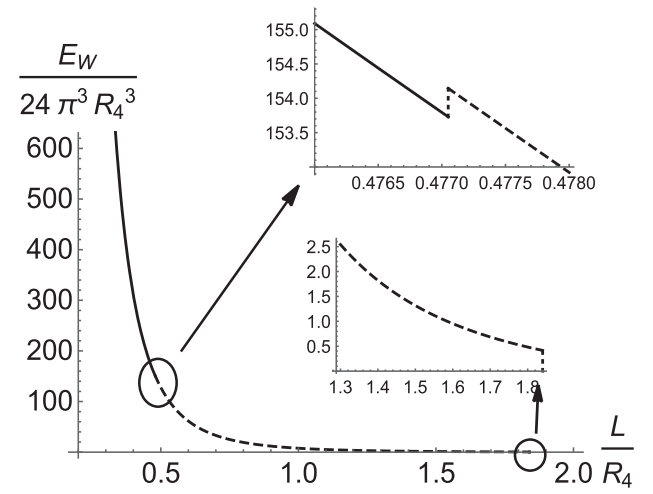

FIG. 10. $E_{W}$ as a function of separation length $L$ along a fixed line $X=0.7 L$. Here solid and dashed lines correspond to $E_{W}$ of the $S_{2}$ and $S_{3}$ phases, respectively.

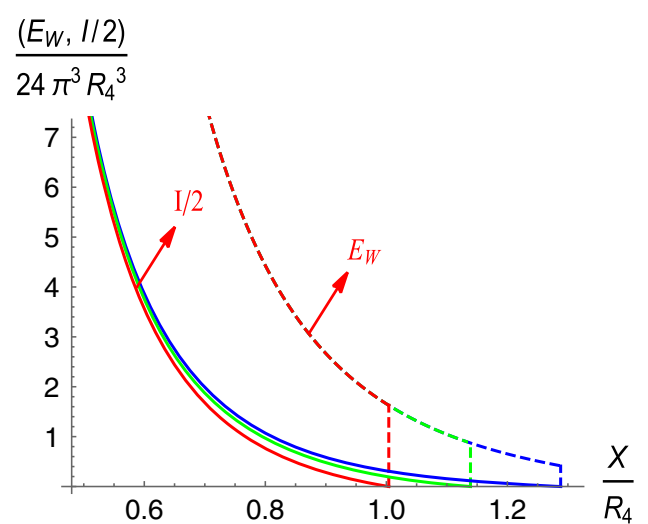

FIG. 11. Mutual information $I$ and entanglement wedge $E_{W}$ as a function of $X$ for different values of $L$. The solid curves correspond to $I / 2$, whereas the dashed curves correspond to $E_{W}$. Here red, green, and blue curves correspond to $L / R_{4}=1.1,1.2$, and 1.3 , respectively.

traced back to the fact that $U_{*}(2 L+X)>U_{0}$ and is also clear from Fig. 5. Therefore, it is clear that $E_{W}$ shows nontrivial features each time a phase transition between different phases occurs.

The entanglement wedge is known to satisfy a few inequalities in holographic settings. In particular, it has been proved that $E_{W}$ always at least exceeds half the mutual information, i.e., $E_{W} \geq I / 2$ [29,86]. In Figs. 11 and 12, we made a comparison between $I / 2$ and $E_{W}$ for diverse values of $X$ and $L$ and find that this inequality is always satisfied in the current holographic confining model. As we will show in later sections, this inequality will remain true for other holographic confining models as well, including a phenomenological bottom-up model. Therefore, the inequality $E_{W} \geq I / 2$ appears to be a generic feature of all holographic models. It would certainly be interesting to see whether such an inequality exists in real QCD or not.

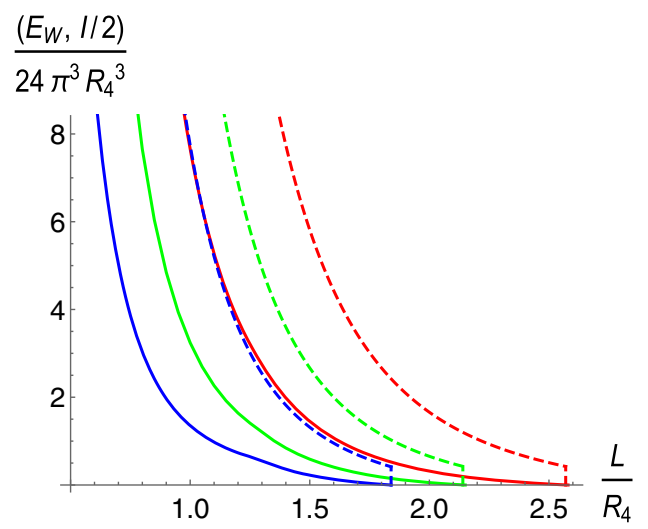

FIG. 12. Mutual information $I$ and entanglement wedge $E_{W}$ as a function of $L$ along a fixed line $X=\alpha L$. The solid curves correspond to $I / 2$, whereas the dashed curves correspond to $E_{W}$. Here red, green, and blue curves correspond to $\alpha=0.5,0.6$, and 0.7 , respectively. 


\section{Entanglement negativity}

We now move on to discuss the entanglement negativity in the current confining theory using the holographic prescription suggested in Refs. [33-35]. For a single interval, the entanglement negativity is given by Eq. (2.16). Note that, in the confined phase where the disconnected surface is more favorable for large strip length, the limiting condition $B \rightarrow A^{c} \rightarrow \infty$ ensures that

$$
\mathcal{A}_{B_{1}}=\mathcal{A}_{B_{2}}=\mathcal{A}_{A \cup B_{1}}=\mathcal{A}_{A \cup B_{2}}=\mathcal{A}_{\text {disconn }},
$$

the above four terms cancel out in Eq. (2.16), and we are left with

$$
\begin{aligned}
\mathcal{E} & =\lim _{B \rightarrow A^{c}} \frac{3}{4}\left[2 S_{A}+S_{B_{1}}+S_{B_{2}}-S_{A \cup B_{1}}-S_{A \cup B_{2}}\right], \\
\mathcal{E} & =\frac{3}{2} S_{A} .
\end{aligned}
$$

Interestingly, the entanglement negativity is just $3 / 2$ times of the entanglement entropy. Since $S_{A}$ depends on $L$ and exhibits a discontinuous behavior at $L_{\text {crit }}$, this discontinuity of $S_{A}$ manifests itself in $\mathcal{E}$ as well. Therefore, interestingly, just like the entanglement entropy, the order of the entanglement negativity also changes from $\mathcal{O}\left(N^{2}\right)$ to $\mathcal{O}\left(N^{0}\right)$ at $L_{\text {crit }}$ :

$$
\begin{array}{ll}
\frac{\partial \mathcal{E}}{\partial L}=\mathcal{O}\left(N^{2}\right) & \text { for } L<L_{\text {crit }} \\
\frac{\partial \mathcal{E}}{\partial L}=\mathcal{O}\left(N^{0}\right) & \text { for } L>L_{\text {crit }}
\end{array}
$$

The discontinuous behavior of the entanglement negativity in the confined phase is a genuine new prediction from holography (strictly speaking, a prediction from the entanglement negativity proposal of Refs. [33-35]) and should be independently tested. Unfortunately, there are no lattice results for the negativity in real QCD to compare with yet. It would really be interesting if this discontinuous nature could be tested via lattice calculations in the near future.

Let us now discuss the entanglement negativity for two disjoint intervals $[39,41]$. Compared to Refs. [39,41], we have $l_{s}=X$ and $l_{1}=l_{2}=L$. So $\mathcal{E}$ is given as

$$
\mathcal{E}=\frac{3}{4}\left[S_{A}(L+X)+S_{A}(L+X)-S_{A}(2 L+X)-S_{A}(X)\right],
$$

where $S_{A}$ is again the entanglement entropy of a single strip of length $L$. Notice that, for $X>L_{\text {crit }}, \mathcal{E}$ is trivially zero, as every term in Eq. (3.17) corresponds to $S_{A}^{\text {discon }}$. This implies that, just like the mutual information and entanglement wedge, $\mathcal{E}$ is zero in the $S_{4}$ phase as well. However, the same is not true for the $S_{1}$ phase. In particular, $\mathcal{E}$ is nonzero and a monotonic function of $X$ and $L$ in the $S_{1}$ phase. This is in

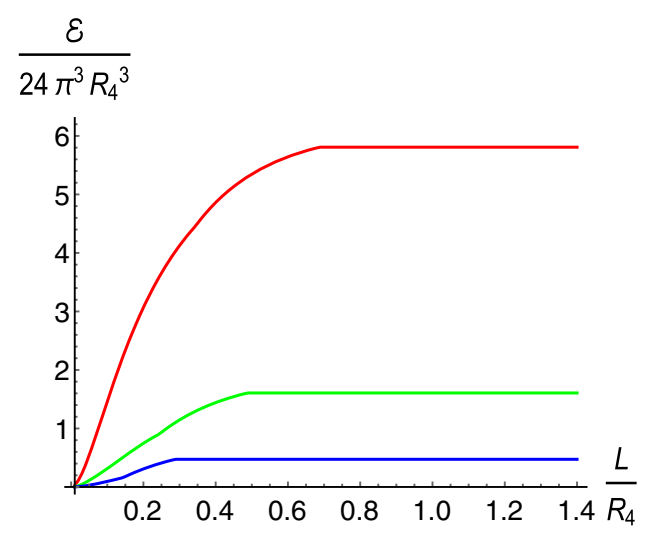

FIG. 13. Entanglement negativity $\mathcal{E}$ of two parallel strips as a function of $L$ for different values of $X$. Here red, green, and blue curves correspond to $X / R_{4}=0.6,0.8$, and 1.0 , respectively.

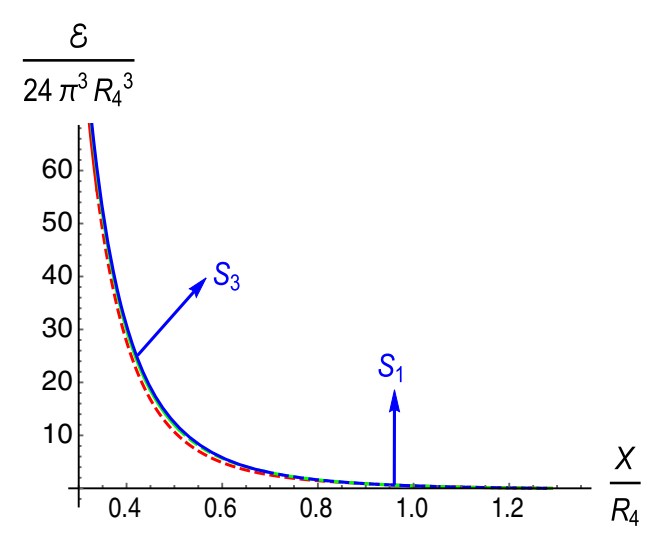

FIG. 14. Entanglement negativity $\mathcal{E}$ of two parallel strips as a function of $X$ for different values of $L$. Solid and dashed lines indicate $\mathcal{E}$ of $S_{3}$ and $S_{1}$ phases, respectively. Here red, green, and blue curves correspond to $L / R_{4}=0.4,0.6$, and 0.8 , respectively.

sharp contrast with the mutual information and entanglement wedge behavior, which are zero in the $S_{1}$ phase as well. The results are shown in Figs. 13 and 14. Note that $\mathcal{E}$ smoothly goes to zero at $X=L_{\text {crit }}$, whereas it remains finite for $L>L_{\text {crit }}$. Moreover, unlike the entanglement wedge, $\mathcal{E}$ is continuous across the various phase transition points but has a cusp (as is visible for Fig. 13). This is again a new prediction form holography and should be tested in lattice settings.

\section{D3 BRANES ON A CIRCLE}

Another top-down confining model can be obtained by wrapping $N_{c} D 3$ branes on a circle of radius $R_{3}$ with twisted boundary conditions for the fermions. Before the wrapping, the low-energy dynamics of $N_{c}$ coincidental $D 3$ branes is given by $\mathcal{N}=4$ supersymmetric Yang-Mills theory with 't Hooft coupling $\lambda=g_{s} N_{c}$. This theory can be reduced to $(2+1)$-dimensional confining theory at long distances by compactifying on a circle. The low-energy 
dynamics of this system is then given by the dimensionless parameter $\lambda_{3}=\lambda / R_{3}$ [70]. In particular, for $\lambda \ll 1$, the theory is described by $(2+1)$-dimensional Yang-Mills theory with coupling $\lambda_{3}$. For $\lambda \gg 1$, on the other hand, one can use the dual gravitational picture in terms of the near-horizon geometry of the $N_{c}$ coincidental $D 3$ branes:

$$
\begin{aligned}
d s^{2}= & \left(\frac{U}{R}\right)^{2}\left[\left(\frac{R}{U}\right)^{4} \frac{d U^{2}}{f(U)}+d x^{\mu} d x_{\mu}\right]+R^{2} d \Omega_{5}^{2} \\
& +\left(\frac{U}{R}\right)^{2} f(U)\left(d x^{3}\right)^{2},
\end{aligned}
$$

where $R$ is the AdS length scale and

$$
f(U)=1-\left(\frac{U_{0}}{U}\right)^{4}, \quad U_{0}^{2}=\frac{\pi \lambda}{R_{3}^{2}}, \quad R^{4}=4 \pi \lambda .
$$

Dilation is a constant, and here we take it to be zero. Note that this geometry again forms a cigar shape in $\left(U, x^{3}\right)$ coordinates, with the radius of the $x^{3}$ circle going to zero as $U \rightarrow U_{0}$.

\section{A. Entanglement entropy: One strip}

The entanglement entropy for this top-down model has also been computed previously in Ref. [9]. Here, we first review their calculation. To compute the entanglement entropy, we consider the previous $D 4$-brane setup by taking a strip subsystem of length $L$, with subsystem domain $-L / 2 \leq x_{1}=x \leq L / 2$ and $0 \leq x_{2} \leq L_{2}$. Using $U=U(x)$, we get the entanglement entropy (2.4) as

$$
S_{A}=\frac{L_{2} \omega_{5}\left(2 \pi R_{3}\right)}{4 G_{N}^{(10)}} \int d x R^{2} U^{3} \sqrt{f(U)+\left(\frac{R}{U}\right)^{4} U^{\prime 2}},
$$

where $\omega_{5}$ is the area of the unit five sphere. In this case also, there are two minimal area surfaces: a $(U$-shaped) connected surface and a disconnected surface. The entanglement entropy of the connected surface turns out to be

$S_{A}^{\text {con }}=\frac{L_{2}}{2 G_{N}^{(10)}}\left(\pi^{4} R^{6}\right) \int_{U_{*}}^{U_{\infty}} d U \frac{U^{4}}{U_{0}} \frac{\sqrt{f(U)}}{\sqrt{U^{6} f(U)-U_{*}^{6} f\left(U_{*}\right)}}$,

where $U_{*}$ is the turning point for the above connected surface and $\left.U^{\prime}(x)\right|_{U=U_{*}}=0$. Furthermore, the strip length $L$ as a function of $U_{*}$ is

$L\left(U_{*}\right)=2 R^{2} \int_{U_{*}}^{U_{\infty}} d U \frac{U_{*}^{3}}{U^{2}} \sqrt{\frac{f\left(U_{*}\right)}{f(U)}} \frac{1}{\sqrt{U^{6} f(U)-U_{*}^{6} f\left(U_{*}\right)}}$,

and the expression of the entanglement entropy of the disconnected surface is

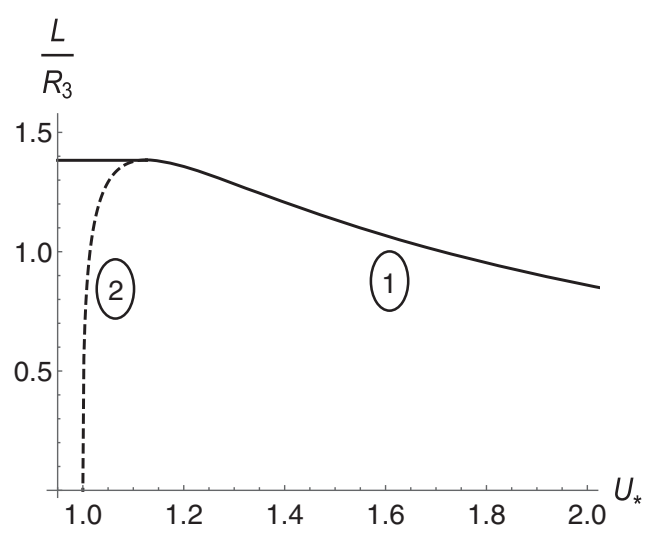

FIG. 15. The behavior of strip length $L$ as a function of $U_{*}$.

$$
S_{A}^{\text {discon }}=\frac{V_{2}}{2 G_{10}}\left(\pi^{4} R^{6}\right) \int_{U_{0}}^{U_{\infty}} d U \frac{U}{U_{0}} .
$$

It is worthwhile to note here that the entanglement entropy of the disconnected surface is again independent of the strip length. This information will be relevant and useful when studying the entanglement structure of the current D3-brane confined setup.

We now show the numerical results of the entanglement entropy in the current confining model. For numerical purpose, we again considered $U_{0}=1$. These numerical results are presented in Figs. 15 and 16, where in the first case we have plotted $L$ as a function of $U_{*}$ and in the second case we have plotted the difference between connected and disconnected entanglement entropies $\left(\triangle S_{A}=S_{A}^{\text {con }}-S_{A}^{\text {discon }}\right)$ as a function of the strip length $L$, respectively. ${ }^{4}$ Note that here also, like in the previous $D 4$-brane model, we have three minimal area surfaces for a given $L$ : one disconnected and two connected. In the plots below, the connected surface (1) (represented by a solid line) is closer to the boundary in comparison to the second connected surface (2) (represented by a dashed line). The second connected surface (2) again corresponds to a saddle point, and its area is always higher than the first connected surface (1). Moreover, the existence of the connected surfaces is dictated by $L_{\max } \simeq 1.383 R_{3}$, as these surfaces can occur only below $L_{\max }$. For values of $L$ beyond $L_{\max }$, we have only the disconnected surface.

We see from Fig. 16 that $\triangle S_{A}$ changes sign with $L$. For small $L, \triangle S_{A}$ is negative. This reflects the fact that for a small subsystem length the connected surface (1) has the smallest area. On the other hand, for large $L, \triangle S_{A}$ is positive, reflecting the fact that for a large subsystem length the disconnected surface has the smallest area. Correspondingly, we have a phase transition at $L=L_{\text {crit }} \simeq$ $1.23 R_{3}$ between connected and disconnected entanglement entropies. As the minimal surface area for the disconnected

\footnotetext{
${ }^{4}$ We have used $L_{2} / 4 G_{N}^{(10)}=1$ in the numerical calculations.
} 


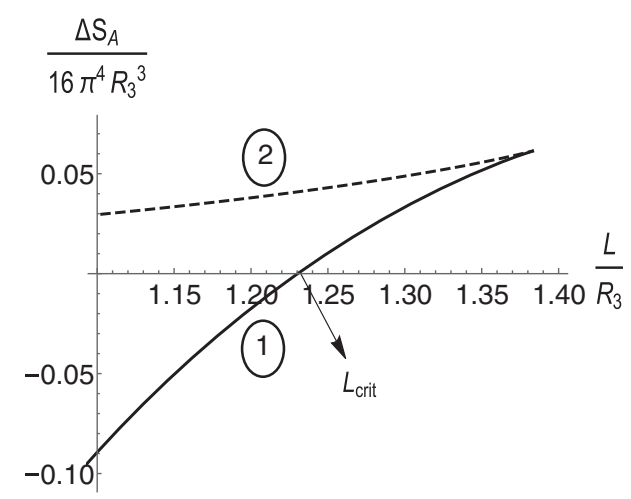

FIG. 16. $\triangle S_{A}=S_{A}^{\text {con }}-S_{A}^{\text {discon }}$ as a function of strip length $L$.

surface is independent of $L$, its entanglement entropy is also independent of $L$. This connected-disconnected phase transition, therefore, leads to the following result (similar to the previous $D 4$-brane setup):

$$
\begin{aligned}
\frac{\partial S_{A}}{\partial L} & \propto \frac{1}{G_{N}^{(10)}}=\mathcal{O}\left(N^{2}\right) \quad \text { for } L<L_{\text {crit }} \\
& \propto \frac{1}{\left[G_{N}^{(10)}\right]^{0}}=\mathcal{O}\left(N^{0}\right) \quad \text { for } L>L_{\text {crit }} .
\end{aligned}
$$

Importantly, once again the order of the entanglement entropy changes as the subsystem size is varied in the confined phase.

\section{B. Mutual information: Two strips}

We now move on to discuss the entanglement structure of two disjoint strips in the current confining system. The two-strip phase diagram of this system has been studied previously in Ref. [55]. Here, we first reproduce their results to set the stage for the discussion of mutual information and entanglement wedge cross section in this system later on. For our purpose, we again consider equal strip lengths, i.e., $\left(L_{1}=L_{2}=L\right)$, for simplicity. This leads to four entangling surfaces $\left\{S_{1}, S_{2}, S_{3}, S_{4}\right\}$, the expressions of which are given in Eq. (3.7). See Fig. 5 for the pictorial representation of these surfaces.

The two-equal-strip entanglement phase diagram is shown in Fig. 17. The four phases $\left\{S_{1}, S_{2}, S_{3}, S_{4}\right\}$ again compete with each other, leading to a similar phase diagram as in the case of $D 4$ branes on a circle confining system. For instance, for small values of $X, L \ll L_{\text {crit }}$, the $S_{1}$ configuration again has the lowest entanglement entropy, whereas for large values of $X, L>L_{\text {crit }}$, it is the $S_{4}$ configuration which has the lowest entanglement entropy. In between, we have $S_{2}$ and $S_{3}$ configurations. The $S_{2}$ to $S_{3}$ phase transition happens at $L=L_{\text {crit }} / 2$ for $X=0$, whereas this phase transition is governed by the equation $2 L+X=L_{\text {crit }}$ for a generic value of $X$.

The two tricritical points, indicated by black dots in Fig. 17, reflect the coexistence of three configurations

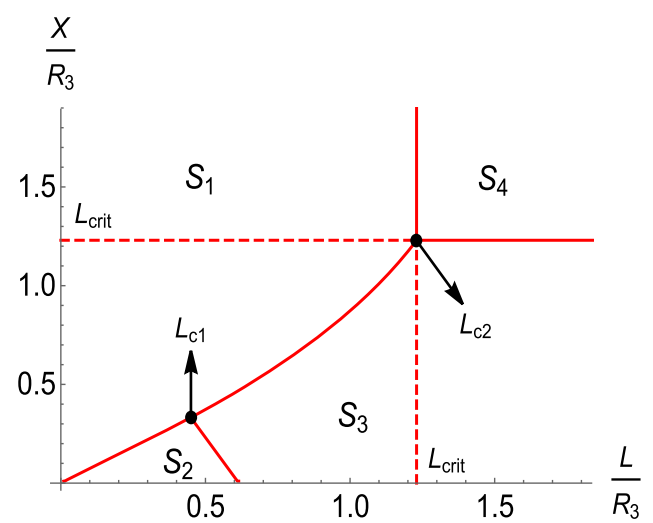

FIG. 17. The entanglement entropy phase diagram of various minimal surfaces for the case of two strips of equal length $L$ separated by a distance $X$ in the confining background of $D 3$ branes on a circle. These four different phases correspond to the four bulk surfaces in Fig. 5 .

together. The first tricritical point $L_{c 1}$ denotes the coexistence of three configurations $\left\{S_{1}, S_{2}, S_{3}\right\}$, and its coordinates are $\left(L / R_{3}=0.45, X / R_{3}=0.331\right)$, whereas the second tricritical point $L_{c 2}$ denotes the coexistence of $\left\{S_{2}, S_{3}, S_{4}\right\}$ configurations, and its coordinates are $\left(L / R_{3}=\right.$ $\left.1.23, X / R_{3}=1.23\right)$. The two-strip phase diagram, along with the presence of tricritical points, again indicates the nonanalytic nature of the entanglement structure in confining theories.

Similarly, one can compute the mutual information in these four phases. The relevant expressions are given in Eq. (3.8). The mutual information is again zero when the two strips have a relatively large separation, i.e., for the $S_{1}$ and the $S_{4}$ configurations, whereas it is nonzero in the remaining $S_{2}$ and $S_{3}$ configurations. The behavior of mutual information as a function of $L$ and $X$ is presented in Figs. 18 and 19. Here, the specific values of $L$ and $X$ are taken in order to move through the $S_{2}$ and $S_{3}$ configurations. It turns out that the mutual information is

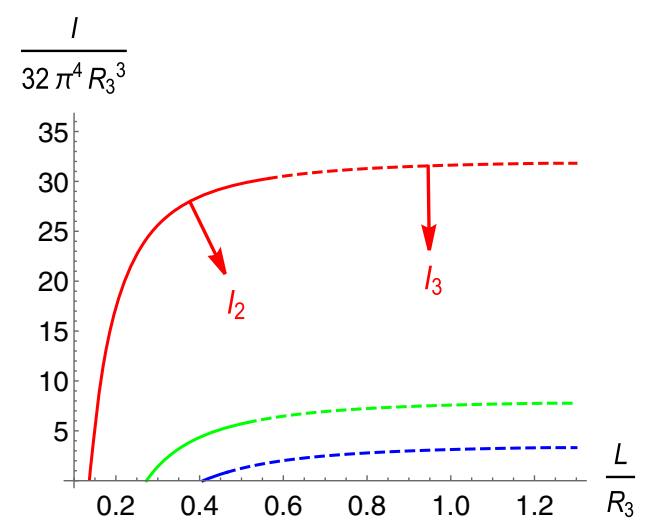

FIG. 18. Mutual information of $S_{2}$ and $S_{3}$ surfaces as a function of $L$. The solid lines correspond to $I_{2}$, whereas the dashed lines correspond to $I_{3}$. The red, green, and blue lines correspond to separation length $X / R_{3}=0.1,0.2$, and 0.3 , respectively. 


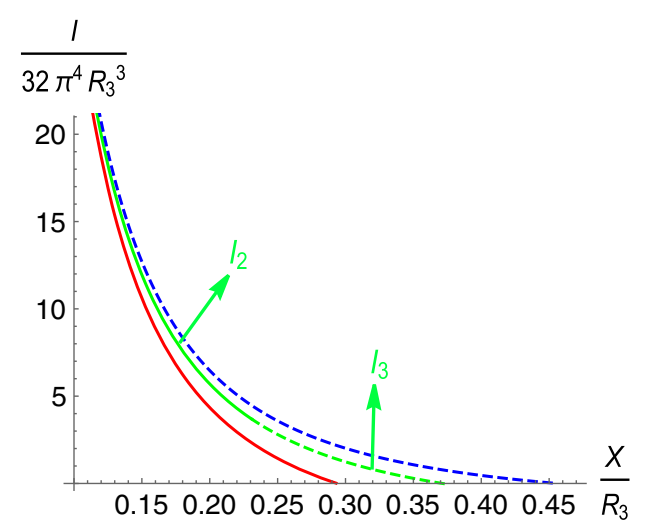

FIG. 19. Mutual information of $S_{2}$ and $S_{3}$ surfaces as a function of $X$. The solid and dashed lines correspond to $I_{2}$ and $I_{3}$, respectively. The red, green, and blue lines correspond to $L / R_{3}=0.4,0.5$, and 0.6 , respectively.

a monotonic function of $L$ and $X$ and varies smoothly as these parameters are changed. Furthermore, the mutual information also exhibits a smooth behavior while passing from one configuration to another. An interesting aspect of this analysis is that there may or may not be a change in the order of mutual information. To be clearer, we see a change in the order of mutual information from $\mathcal{O}\left(N^{2}\right)$ to $\mathcal{O}\left(N^{0}\right)$ as we move from $S_{2}$ to $S_{1}$ by varying $X$. On the other hand, there is no such change in the order as we move from $S_{2}$ to $S_{3}$ by varying $L$. Therefore, we again have the following relations in the current confined phase:

$\begin{array}{lll}\frac{\partial I_{1}}{\partial L} \propto \frac{1}{G_{N}^{0}}=\mathcal{O}\left(N^{0}\right), & \frac{\partial I_{2}}{\partial L} \propto \frac{1}{G_{N}}=\mathcal{O}\left(N^{2}\right), \\ \frac{\partial I_{3}}{\partial L} \propto \frac{1}{G_{N}}=\mathcal{O}\left(N^{2}\right), & \frac{\partial I_{4}}{\partial L} \propto \frac{1}{G_{N}^{0}}=\mathcal{O}\left(N^{0}\right) .\end{array}$

\section{Entanglement wedge cross section}

Similar to the previous $D 4$-brane setup, the symmetry of the strip configuration again dictates that the entanglement wedge cross section in the current confining background is given by the area of a constant- $x$ hypersurface $\Sigma$, located in the middle of the strips (as depicted in Fig. 5). From the following induced metric on $\Sigma$ :

$$
\begin{aligned}
\left(d s^{2}\right)_{\Sigma}^{\text {ind }}= & \left(\frac{U}{R}\right)^{2}\left[\left(\frac{R}{U}\right)^{4} \frac{d U^{2}}{f(U)}+d x^{2} d x_{2}\right] \\
& +R^{2} d \Omega_{5}^{2}+\left(\frac{U}{R}\right)^{2} f(U)\left(d x^{3}\right)^{2},
\end{aligned}
$$

we can get the expression of the entanglement wedge cross section as

$$
E_{W}=\frac{1}{4 G_{10}} \int\left(d^{8} \sigma\right)\left(e^{-2 \phi}\right) \sqrt{g_{\Sigma}^{\text {ind }}} .
$$

When considering the two-disjoint-strip setup, similar to the previous $D 4$-brane case, the entanglement wedge cross section is zero for the $S_{1}$ and $S_{4}$ configurations, while it is nonzero for the $S_{2}$ and $S_{3}$ configurations. In particular, for the $S_{2}$ configuration it is given by

$$
\begin{aligned}
E_{W}^{2} & =\frac{L_{2}}{4 G_{10}}\left(\pi^{4} R^{6}\right) \int_{U_{*}(2 l+x)}^{U_{*}(x)} d U \frac{U}{U_{0}} \\
& =\frac{L_{2}}{4 G_{10}}\left(\frac{\pi^{4} R^{6}}{2 U_{0}}\right)\left[U_{*}^{2}(x)-U_{*}^{2}(2 l+x)\right],
\end{aligned}
$$

whereas for the $S_{3}$ configuration it is given by

$$
\begin{aligned}
E_{W}^{3} & =\frac{L_{2}}{4 G_{10}}\left(\pi^{4} R^{6}\right) \int_{U_{0}}^{U_{*}(x)} d U \frac{U}{U_{0}} \\
& =\frac{L_{2}}{4 G_{10}}\left(\frac{\pi^{4} R^{6}}{2 U_{0}}\right)\left[U_{*}^{2}(x)-U_{0}^{2}\right] .
\end{aligned}
$$

From the above equations, one can again conclude that both $E_{W}^{2}$ and $E_{W}^{3}$ are positive, as $U_{*}(x) \geq U_{*}(2 L+X) \geq U_{0}$.

The behavior of the entanglement wedge as a function of $X$ for specific values of $L$ is shown in Fig. 20. These specific values of $L$ are again chosen so that we can see the nature of $E_{W}$ close to the $S_{1} / S_{3}$ and $S_{3} / S_{4}$ phase transition points. From this analysis we see that $E_{W}$ changes monotonically with respect to $X$ and shows discontinuity at the transition point. The discontinuity in $E_{W}$ at the $S_{3} / S_{4}$ transition line can also be inferred from Eq. (4.12). In particular, the condition $U_{*}\left(X=L_{\text {crit }}\right)>U_{0}$ ensures that $E_{W}^{3}>0$ at $X_{\text {crit }}$ (as opposed to $E_{W}^{4}$, which is zero at $X_{\text {crit }}$ ). In the same fashion, $E_{W}^{3}$ picks up a nonzero value at the $S_{1} / S_{3}$ transition line (as indicated by a red curve). Our entire exercise suggests that the entanglement wedge cross section disappears in a discontinuous fashion when the values of $X$ and $L$ are large in the case of confining backgrounds.

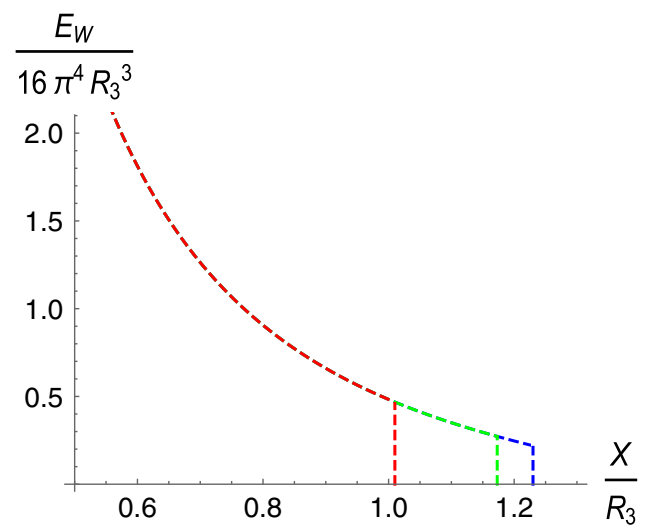

FIG. 20. $E_{W}$ as a function of separation length $X$ for different values of strip length $L$. Here blue, green, and red curves correspond to $L / R_{3}=1.3>L_{\text {crit }}, 1.2$, and 1.1, respectively. 


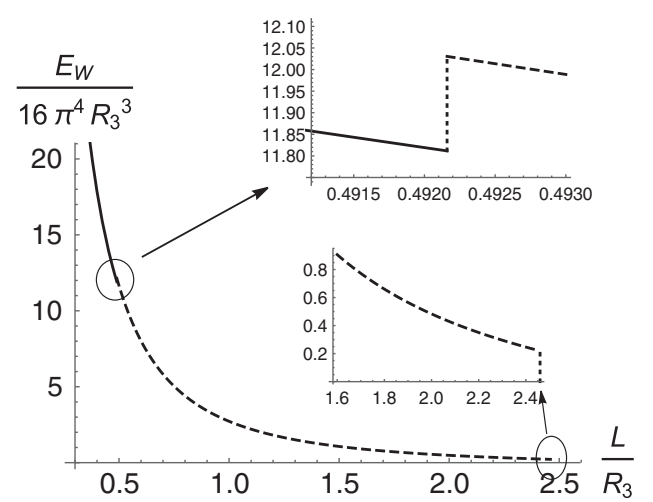

FIG. 21. $\quad E_{W}$ as a function of separation length $L$ along a fixed line $X=0.5 L$. Here solid and dashed lines correspond to $E_{W}$ of $S_{2}$ and $S_{3}$ phases, respectively.

We have also studied the nature of $E_{W}$ close to the $S_{2} / S_{3}$ critical line. This analysis is shown in Fig. 21. We have chosen a specific value of $X=0.5 \mathrm{~L}$ in order to study $E_{W}$ for $S_{2}, S_{3}$, and $S_{4}$ configurations simultaneously. We find a discontinuity in $E_{W}$ at the $S_{2} / S_{3}$ transition line as well. This discontinuous behavior can also be inferred from Eqs. (4.11) and (4.12). In particular, the condition $U_{*}(2 L+X) \neq U_{0}$ ascertains that $E_{W}^{2}$ and $E_{W}^{3}$ cannot have the same value at the $S_{2} / S_{3}$ critical point. There is also a positive jump in the value of $E_{W}$ as the $S_{2} / S_{3}$ critical point is approached from the $S_{2}$ side, indicating an increase in the area of entanglement wedge at the critical point. This increment in area can also be extracted from the condition $U_{*}(2 L+X)>U_{0}$. To conclude, we see that $E_{W}$ shows a nontrivial behavior whenever a phase transition happens.

We further tested the inequality $E_{W} \geq I / 2$ in the current confining setup. We have studied this inequality numerically for various values of $X$ and $L$ and found it to be always true. The results for this exercise are presented in Figs. 22 and 23.

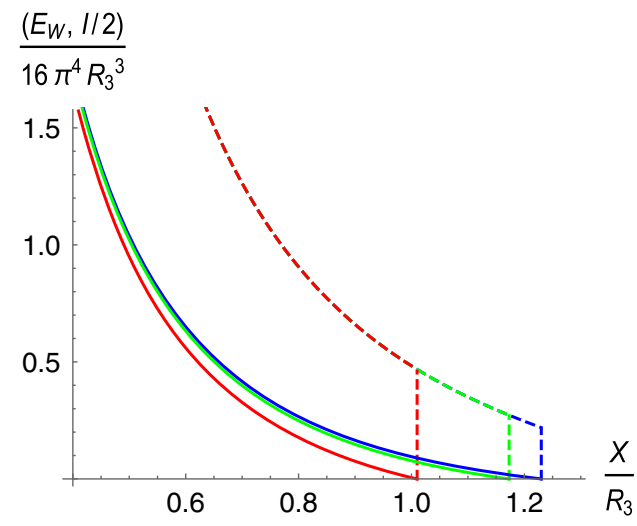

FIG. 22. Mutual information $I$ and entanglement wedge $E_{W}$ as a function of $X$ for different values of $L$. The solid curves correspond to $I / 2$, whereas the dashed curves correspond to $E_{W}$. Here red, green, and blue curves correspond to $L / R_{3}=1.3,1.2$, and 1.1 , respectively.

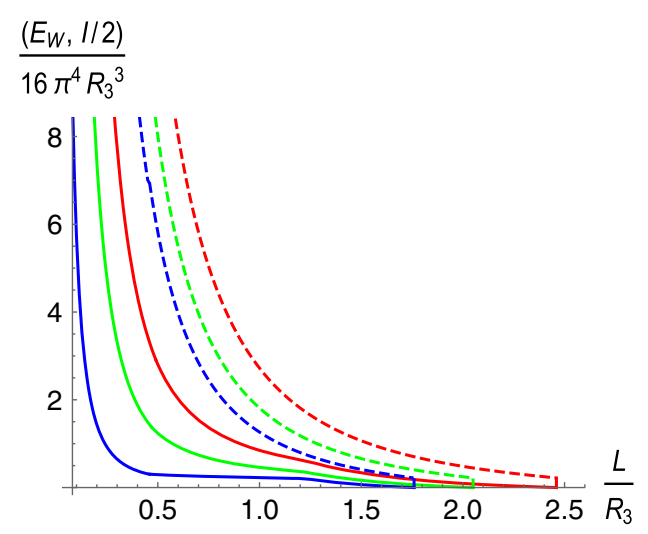

FIG. 23. Mutual information $I$ and entanglement wedge $E_{W}$ as a function of $L$ along a fixed line $X=\alpha L$. The solid curves correspond to $I / 2$, whereas the dashed curves correspond to $E_{W}$. Here red, green, and blue curves correspond to $\alpha=0.5,0.6$, and 0.7 , respectively.

\section{Entanglement negativity}

The holographic entanglement negativity for a single interval is given by Eq. (2.16). In the limit $B \rightarrow A^{c} \rightarrow \infty$, we again have

$$
\mathcal{A}_{B_{1}}=\mathcal{A}_{B_{2}}=\mathcal{A}_{A \cup B_{1}}=\mathcal{A}_{A \cup B_{2}}=\mathcal{A}_{\text {disconn }},
$$

which leads to the following result for the negativity:

$\mathcal{E}=\lim _{B \rightarrow A^{c}} \frac{3}{4}\left[2 S_{A}+S_{B_{1}}+S_{B_{2}}-S_{A \cup B_{1}}-S_{A \cup B_{2}}\right]$,

$\mathcal{E}=\frac{3}{2} S_{A}$.

This again tells us that the entanglement negativity is discontinuous at $L_{\text {crit }}$. Consequently, in this confining model as well, a change in the order of entanglement negativity [from $\mathcal{O}\left(N^{2}\right)$ to $\mathcal{O}\left(N^{0}\right)$ or vice versa] appears at $L_{\text {crit }}$.

The entanglement negativity for the two disjoint strips is again given by $[39,41]$

$\mathcal{E}=\frac{3}{4}\left[S_{A}(L+X)+S_{A}(L+X)-S_{A}(2 L+X)-S_{A}(X)\right]$,

where $S_{A}$ is the entanglement entropy of a single interval. For the $S_{4}$ configuration, where $X>L_{\text {crit }}, \mathcal{E}$ is zero, as all the terms in Eq. (4.15) become $S_{A}^{\text {discon }}$, whereas $\mathcal{E}$ is nonzero in all other configurations, in particular, in the $S_{1}$ configuration. This behavior is different from the mutual information and entanglement wedge cross section behavior, which were in the $S_{1}$ configuration as well. From Figs. 24 and 25, we can infer that $\mathcal{E}$, in general (except for the $S_{4}$ case), has a nonzero value and behaves monotonically with respect to $X$ and $L$. Furthermore, $\mathcal{E}$ is finite for $L>L_{\text {crit }}$, and it goes to 


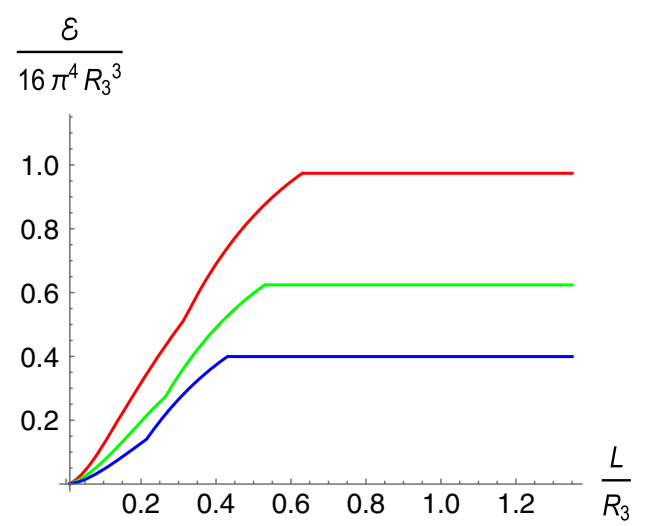

FIG. 24. Entanglement negativity $\mathcal{E}$ of two parallel strips as a function of $X$ for different values of $L$. Here red, green, and blue curves correspond to $X / R_{3}=0.6,0.7$, and 0.8 , respectively.

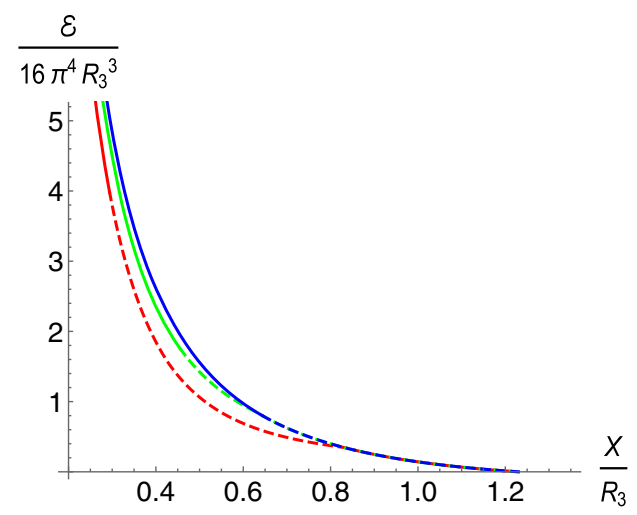

FIG. 25. Entanglement negativity $\mathcal{E}$ of two parallel strips as a function of $L$ for different values of $X$. Here red, green, and blue curves correspond to $L / R_{4}=0.4,0.6$, and 0.8 , respectively.

zero in a smooth fashion at $X=L_{\text {crit }}$. In contrast to the entanglement wedge, $\mathcal{E}$ shows a continuous behavior during the phase transition but exhibits a cusp (see Fig. 24).

Before we end our discussion on top-down models, we further add that we have performed similar analysis in other top-down confining models as well. In particular, we have also computed entanglement entropy, mutual information, entanglement wedge cross section, and entanglement negativity in the Klebanov-Strassler and KlebanovTseytlin confining backgrounds [116], and results similar to those presented here are found for these entanglement measures.

\section{BOTTOM-UP HOLOGRAPHIC CONFINING MODEL}

Having discussed the entanglement measures in topdown holographic confining models, we now move on to discuss them in a bottom-up confining model. As is well known, the top-down holographic QCD models generally exhibit undesirable features whose analogs in real QCD do not exist. In particular, the dual boundary theory of these top-down holographic models usually contains nonrunning coupling constants, additional Hilbert states (coming from the Kaluza-Klein modes of extra dimensions), problematic conformal symmetries, etc., whereas the phenomenological bottom-up holographic models, although they lack strong AdS/CFT justification and generally constitute in an ad hoc manner to reproduced desirable QCD-like features of the dual boundary theory, can overcome most of the difficulties of the top-down models. Therefore, it is interesting to see how different entanglement measures behave in bottom-up confining models as well.

Here, we consider a particular bottom-up confining model suggested in Refs. [51,71]. This model is based on the Einstein-Maxwell-dilaton (EMD) gravity action. Importantly, this gravity model can be solved exactly, and the closed loop expressions of the spacetime metric can be found. ${ }^{5}$ Furthermore, this model predicts a thermal-AdSblack hole phase transition, which on the dual boundary theory corresponds to the confined-deconfined phase transition. The model, moreover, exhibits a linear Regge trajectory for a heavy meson spectrum. Here, we briefly describe the analytic expression of the relevant spacetime metric, which will be needed for our discussion in later sections, and more details can be found in Refs. [51,71].

In this model, the dual spacetime geometry for the confined phase is

$d s^{2}=\frac{R^{2} e^{2 A(z)}}{z^{2}}\left(-d t^{2}+d z^{2}+d y_{1}^{2}+d y_{2}^{2}+d y_{3}^{2}\right)$

where $A(z)=-a z^{2}$, with $a>0$, is the scale function and $R$ is the AdS length scale. The radial coordinate $z$ runs from $z=0$ (asymptotic boundary) to $z=\infty$ (deep bulk). This solution asymptotes to AdS near the boundary $(z \rightarrow 0)$ and has a negative curvature throughout the spacetime. The parameter $a=0.145 \mathrm{GeV}^{2}$ is fixed by demanding the thermal-AdS-black hole (or the dual confined-deconfined) phase transition to be around $270 \mathrm{MeV}$, as is observed in large- $N$ lattice QCD in the pure glue sector.

\section{A. Entanglement entropy: One strip}

The behavior of entanglement entropy in this confining model has already been studied in Refs. [51,52]. Here, we briefly mention their results. For a strip subsystem $A$ of domain $\quad\left\{-L / 2 \leq y_{1} \leq L / 2,0 \leq y_{2} \leq L_{2}, 0 \leq y_{3} \leq L_{3}\right\}$, the entanglement entropy of the connected surface is given by

\footnotetext{
${ }^{5}$ Analytical solutions can be found for the EMD gravity system using the potential reconstruction method. For more details on this method, see Refs. [117,118].
} 


$$
S_{A}^{\mathrm{con}}(L)=\frac{L_{2} L_{2} R^{3}}{4 G_{N}^{(5)}} \int_{0}^{z_{*}} d z \frac{2 z_{*}^{3}}{z^{3}} \frac{e^{3 A(z)-3 A\left(z_{*}\right)}}{\sqrt{\left[z_{*}^{6} e^{-6 A\left(z_{*}\right)}-z^{6} e^{-6 A(z)}\right]}},
$$

where $z_{*}$ is the turning point of the connected minimal area surface and is related to the strip length $L$ in the following way:

$$
L=2 \int_{0}^{z_{*}} d z \frac{z^{3} e^{-3 A(z)}}{\sqrt{\left[z_{*}^{6} e^{-6 A\left(z_{*}\right)}-z^{6} e^{-A(z)}\right]}}
$$

whereas the entanglement entropy for the disconnected surface is given by

$$
S_{A}^{\text {discon }}=\frac{L_{2} L_{3} R^{3}}{4 G_{N}^{(5)}} \int_{0}^{\infty} d z \frac{2 e^{3 A(z)}}{z^{3}} .
$$

Note that the entanglement entropy of the disconnected surface is again independent of the strip length $L$.

The numerical results for the entanglement entropy are shown in Figs. 26 and $27 .{ }^{6}$ Similar to the top-down confining models, here again, a maximum length appears $\left(L_{\max } \simeq 0.959\right)$ above which no solution for the connected surface exists. Below $L_{\max }$, there are two solutions for the connected surface, from which the surface which is nearer to the boundary always has the smallest area (shown by a solid line). The entanglement entropy again undergoes a phase transition from connected to disconnected surface as $L$ increases. This transition happens at $L_{\text {crit }} \simeq 0.951<L_{\max }$, at which the area of the connected surface becomes larger than the disconnected surface. Correspondingly, in this bottomup confining system as well, the entanglement entropy becomes independent of $L$ for large $L$. The discontinuous nature of the entanglement entropy, therefore, appears to be a generic feature of all holographic confining theories.

\section{B. Mutual information: Two strips}

The two-equal-strip phase diagram of the EMD bottomup confining background is shown in Fig. 28. ${ }^{7}$ Its two-strip entanglement phase structure is quite similar to the topdown confining models. In particular, depending upon the magnitude of $X$ and $L$, four different entangling surfaces $\left\{S_{1}, S_{2}, S_{3}, S_{4}\right\}$ again dominate the entanglement structure. The expressions of $\left\{S_{1}, S_{2}, S_{3}, S_{4}\right\}$ are the same as in Eq. (3.7).

${ }^{6}$ Here, and in the subsequent subsections, we have used $\frac{L_{2} L_{3} R^{3}}{4 G_{N}^{(5)}}=1$ for numerical purposes.

${ }^{7}$ The two-equal-strip entanglement phase diagram and mutual information of this bottom-up confined system have also been investigated in Ref. [52].

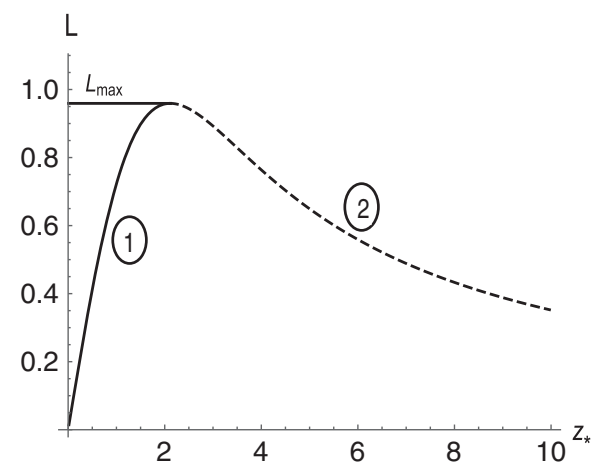

FIG. 26. The behavior of strip length $L$ as a function of $z_{*}$.

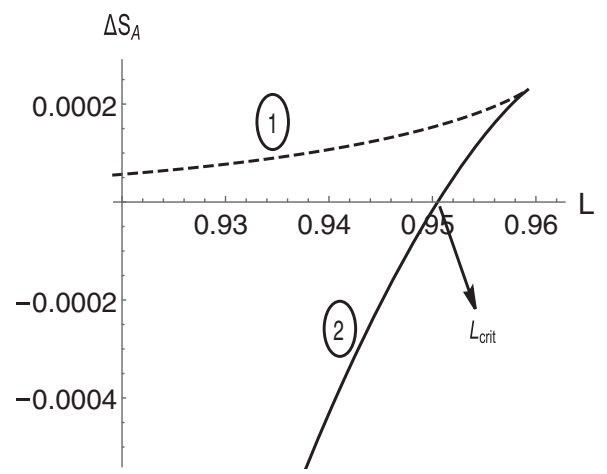

FIG. 27. $\triangle S_{A}=S_{A}^{\text {con }}-S_{A}^{\text {discon }}$ as a function of strip length $L$.

There are again two tricritical points, where three phases coexist. The coordinates of these tricritical points are $(L=0.343, X=0.266)$ and $(L=0.951, X=0.951)$. These tricritical points, as well as other critical lines, again suggest various nonanalyticities in the entanglement structure of the confined phase.

Similarly, the mutual information in these four entangling phases is given by Eq. (3.8). It again goes to zero in $S_{1}$ and $S_{4}$ phases, whereas it remain finite and positive for $S_{2}$ and $S_{3}$ phases. Therefore, the mutual information is again

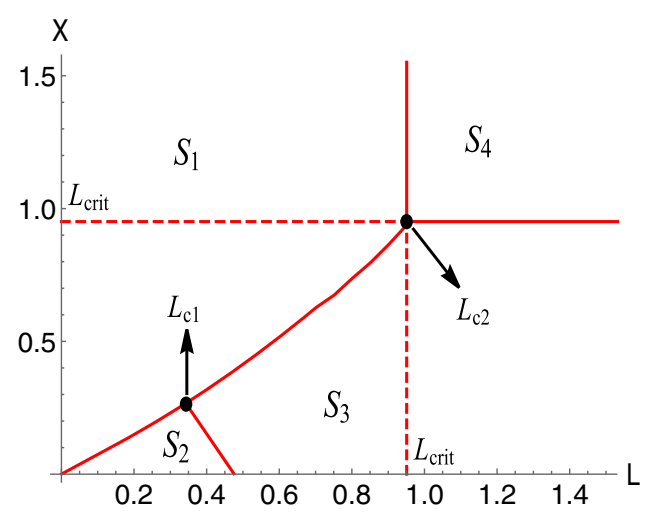

FIG. 28. The two-equal-strip entanglement phase diagram for the EMD bottom-up confining background. The four different phases correspond to the four bulk surfaces in Fig. 5. Two black dots indicate the two tricritical points. 


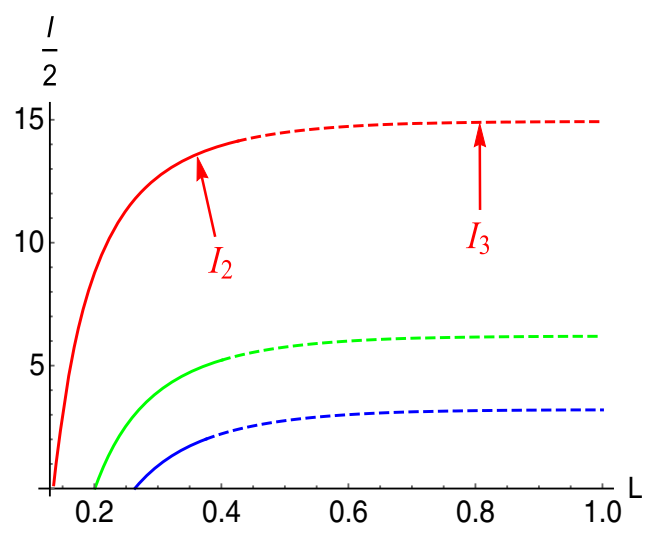

FIG. 29. Mutual information of $S_{2}$ and $S_{3}$ phases as a function of $L$. The solid and dashed lines correspond to $I_{2}$ and $I_{3}$, respectively. The red, green, and blue lines correspond to $X=0.10,0.15$, and 0.20 , respectively.

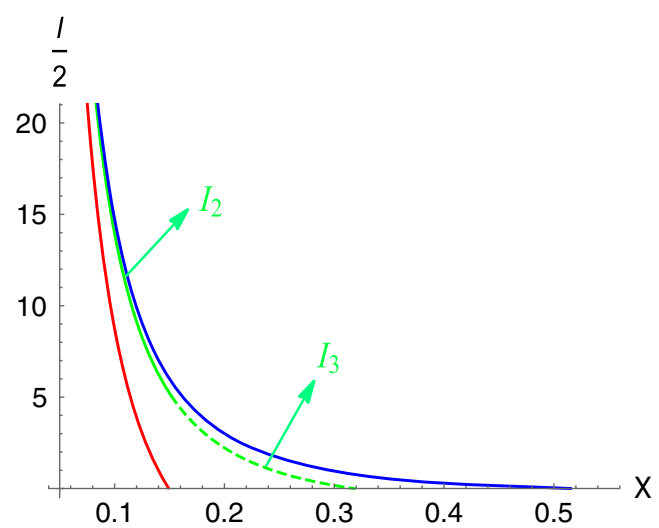

FIG. 30. Mutual information of $S_{2}$ and $S_{3}$ phases as a function of $X$. The solid and dashed lines correspond to $I_{2}$ and $I_{3}$, respectively. The red, green, and blue lines correspond to $L=0.2,0.4$, and 0.6 , respectively.

of the order of $\mathcal{O}\left(N^{2}\right)$ in $S_{2}$ and $S_{3}$ phases, whereas it is of the order of $\mathcal{O}\left(N^{0}\right)$ in $S_{1}$ and $S_{4}$ phases. In Figs. 29 and 30, we have shown the variation of mutual information in $S_{2}$ and $S_{3}$ phases as a function of $X$ and $L$. It again connects smoothly between $S_{2}$ and $S_{3}$ phases. Moreover, as we approach $S_{1}$ and $S_{4}$ phases from $S_{2}$ and $S_{3}$ phases by changing $X$ and $L$, it again goes to zero in a continuous manner. It is clear that, apart from a qualitative change in the magnitude of the critical points, the overall mutual information structure of this bottom-up EMD confining model remains the same as in the above discussed topdown confining models.

\section{Entanglement wedge cross section}

We now calculate the entanglement wedge cross section of the dual confining theory of Eq. (5.5). It is given by the minimal area of $t=$ const and $y_{1}=$ const surface. The induced metric on this surface is

$$
d s^{2}=\frac{R^{2} e^{2 A(z)}}{z^{2}}\left(d z^{2}+d y_{2}^{2}+d y_{3}^{2}\right)
$$

which implies

$$
E_{W}=\frac{L_{2} L_{3} R^{3}}{4 G_{N}^{(5)}} \int d z \frac{e^{-3 A(z)}}{z^{3}}
$$

As earlier, out of the four surfaces, the disjoint surfaces $S_{1}$ and $S_{4}$ again have zero entanglement wedge cross section, whereas the connected surfaces $S_{2}$ and $S_{3}$ have a nonzero entanglement wedge cross section. For the $S_{2}$ phase, it is given by

$$
\begin{aligned}
E_{W}^{2} & =\frac{L_{2} L_{3} R^{3}}{4 G_{N}^{(5)}} \int_{z_{*}(X)}^{z_{*}(2 L+X)} d z \frac{e^{-3 A(z)}}{z^{3}} \\
& =-\frac{L_{2} L_{3} R^{3}}{4 G_{N}^{(5)}}\left|\frac{e^{-3 a z^{2}}}{2 z^{2}}+\frac{3 a}{2} E i\left(-3 a z^{2}\right)\right|_{z_{*}(X)}^{z_{*}(2 L+X)},
\end{aligned}
$$

where $E i(x)$ is an exponential integral function. Similarly, for the $S_{3}$ phase,

$$
\begin{aligned}
E_{W}^{3} & =\frac{L_{2} L_{3} R^{3}}{4 G_{N}^{(5)}} \int_{z_{*}(X)}^{\infty} d z \frac{e^{-3 A(z)}}{z^{3}} \\
& =\frac{L_{2} L_{3} R^{3}}{4 G_{N}^{(5)}}\left[\frac{e^{-3 a z_{*}^{2}(X)}}{2 z_{*}^{2}(X)}-3 a \Gamma\left[0,3 a z_{*}^{2}(X)\right]\right],
\end{aligned}
$$

where $\Gamma[a, b]$ is the incomplete gamma function. Note that $E_{W}^{3}$ is actually independent of strip length $L$ and depends only on $X$. This would imply that $E_{W}$ profiles for different values of $L$ will overlap in the $S_{3}$ phase. Also, from the definition itself, it is explicitly clear that both $E_{W}^{2}$ and $E_{W}^{3}$ are positive.

In Fig. 31, the variation of $E_{W}$ as a function of $X$ is shown. We find that $E_{W}$, again, is not only a monotonically

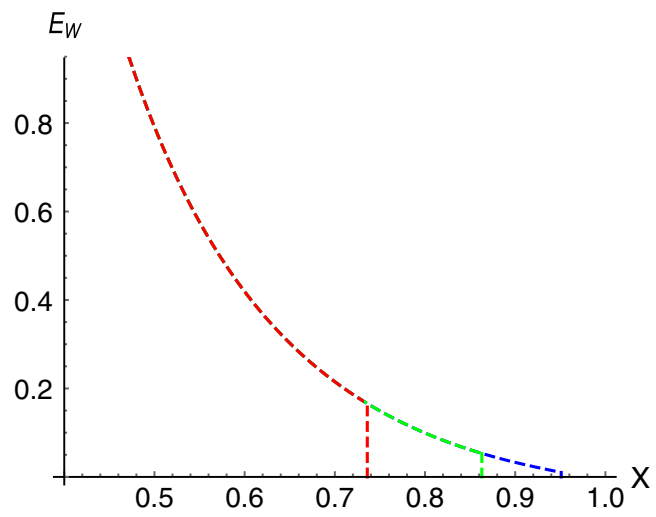

FIG. 31. $E_{W}$ as a function of $X$ for different values of $L$. Here red, green, and blue curves correspond to $L=0.8,0.9$, and $1.0>L_{\text {crit }}$, respectively. 


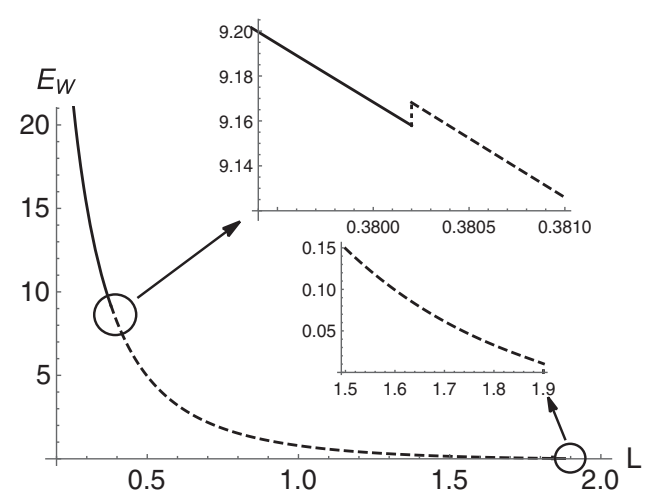

FIG. 32. $E_{W}$ as a function of $L$ along a fixed line $X=0.5 L$. Here solid and dashed lines correspond to $E_{W}$ of $S_{2}$ and $S_{3}$ phases, respectively.

decreasing function of $X$, but also discontinuous at the critical points. In particular, $E_{W}$ does not go to zero as the $S_{1} / S_{3}$ and $S_{3} / S_{4}$ critical lines are approached from the $S_{3}$ side. Therefore, just like in the top-down confining models, the entanglement wedge also vanishes discontinuously for large values of $X$ and $L$ in the EMD confining model.

To further explore the discontinuous nature of $E_{W}$, we investigate its behavior near the $S_{2} / S_{3}$ critical points. This is interesting considering that $E_{W}$ is nonzero in both these phases. The results are shown in Fig. 32. Here $E_{W}$ is evaluated along a particular line $X=0.5 \mathrm{~L}$, which allows us probe its behavior in three different phases $\left\{S_{2}, S_{3}, S_{4}\right\}$ simultaneously. We again find a discontinuous jump in the area of the entanglement wedge at the $S_{2} / S_{3}$ critical point. In particular, $E_{W}^{2}$ and $E_{W}^{3}$ values do not match at the $S_{2} / S_{3}$ critical point. We checked this behavior for many other $X=\alpha L$ lines and found a similar discontinuous pattern at the $S_{2} / S_{3}$ critical points. Moreover, as also mentioned above, $E_{W}$ again exhibited a discontinuous pattern at the $S_{3} / S_{4}$ critical points. Since a similar kind of results

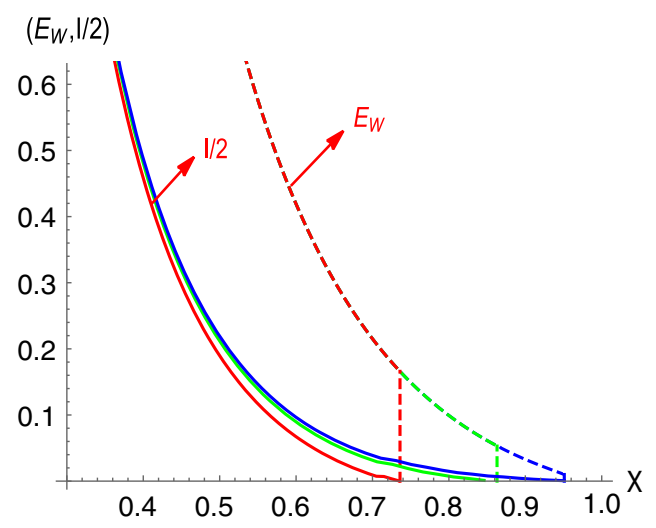

FIG. 33. Mutual information $I$ and entanglement wedge $E_{W}$ as a function of $X$ for different values of $L$. The solid curves correspond to $I / 2$, whereas the dashed curves correspond to $E_{W}$. Here red, green, and blue curves correspond to $L=0.8,0.9$, and 1.0 , respectively.

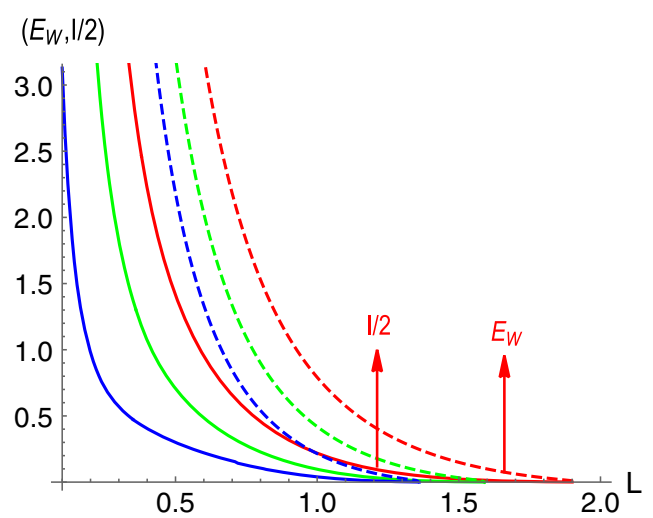

FIG. 34. Mutual information $I$ and entanglement wedge $E_{W}$ as a function of $L$ along a fixed line $X=\alpha L$. The solid curves correspond to $I / 2$, whereas the dashed curves correspond to $E_{W}$. Here red, green, and blue curves correspond to $\alpha=0.5,0.6$, and 0.7 , respectively.

appeared in the top-down confining models as well, these findings advocate for the case that the nonanalyticity in the structure of $E_{W}$ is a universal feature of all holographic confining theories.

We further tested the entanglement wedge and mutual information inequality $\left(E_{W} \geq I / 2\right)$ in the current EMD confining model. The results are shown in Figs. 33 and 34. We find that this inequality is again satisfied. We have numerically checked this inequality for many different values of $X$ and $L$ and find that $E_{W}$ is always greater than $I / 2$. The inequality saturates only at the critical points, at which $I / 2$ continuously goes to zero, whereas $E_{W}$ exhibits a sharp drop to zero.

\section{Entanglement negativity}

We now calculate the entanglement negativity in the current EMD confining model. For a single interval subsystem, it is given by Eq. (2.16). Since the disconnected entanglement entropy, which is independent of the strip length, is more favorable at large strip lengths in this confining model as well, the limiting condition $B \rightarrow A^{c} \rightarrow \infty$ again ensures that

$$
S_{B_{1}}=S_{B_{2}}=S_{A \cup B_{1}}=S_{A \cup B_{2}}=S_{A}^{\text {discon }} .
$$

This implies

$\mathcal{E}=\lim _{B \rightarrow A^{c}} \frac{3}{4}\left[2 S_{A}+S_{B_{1}}+S_{B_{2}}-S_{A \cup B_{1}}-S_{A \cup B_{2}}\right]$,

$\mathcal{E}=\frac{3}{2} S_{A}$.

Again, the entanglement negativity is just $3 / 2$ times of the entanglement entropy. Therefore, the discontinuous nature of the entanglement entropy at $L_{\text {crit }}$ again implies a nonanalytic behavior of the entanglement negativity at 
$\varepsilon$

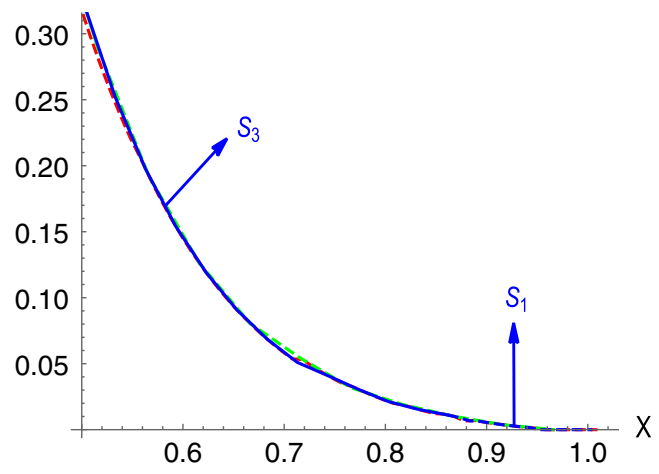

FIG. 35. Entanglement negativity $\mathcal{E}$ of two parallel strips as a function of $X$ for different values of $L$. Solid and dashed lines indicate $\mathcal{E}$ of $S_{3}$ and $S_{1}$ phases, respectively. Here red, green,l and blue curves correspond to $L=0.4,0.6$, and 0.8 , respectively.

the same critical length. Correspondingly, the entanglement negativity exhibits an order change [from $\mathcal{O}\left(N^{2}\right)$ to $\mathcal{O}\left(N^{0}\right)$ or vice versa] at $L_{\text {crit }}$ in this confining background as well.

For the two disjoint equal strip intervals, the entanglement negativity is given by Eq. (3.17):

$\mathcal{E}=\frac{3}{4}\left[S_{A}(L+X)+S_{A}(L+X)-S_{A}(2 L+X)-S_{A}(X)\right]$.

The numerical results of the entanglement negativity for two strips are shown in Figs. 35 and 36. It again turns out to be a monotonic function of $X$ and $L$. Notably, unlike the entanglement wedge and mutual information, it remains nonzero in the $S_{1}$ phase. In particular, the entanglement negativity is zero only when the separation between the two strips is larger than $L_{\text {crit }}$. This also implies entanglement negativity is trivially zero in the $S_{4}$ phase (i.e., $X>L_{\text {crit }}$, $L>L_{\text {crit }}$ ). In the $S_{2}$ and $S_{3}$ phases, entanglement negativity is finite and positive. Importantly, the entanglement

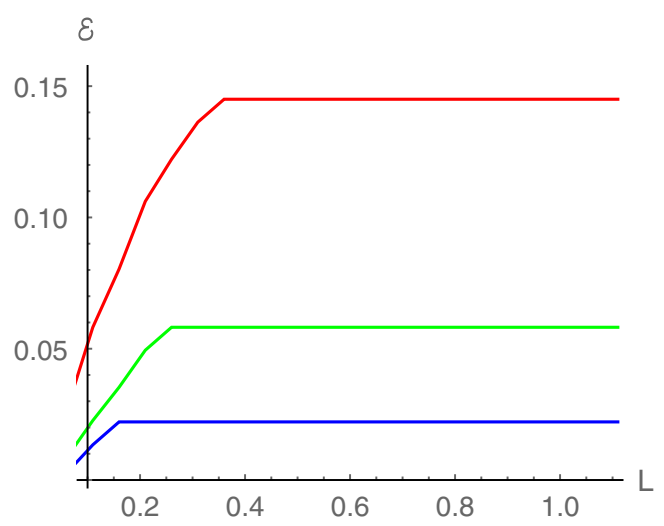

FIG. 36. Entanglement negativity $\mathcal{E}$ of two parallel strips as a function of $L$ for different values of $X$. Here red, green, and blue curves correspond to $X=0.6,0.7$, and 0.8 , respectively. negativity remains continuous across the various phase transitions (though again exhibits a cusp), and it smoothly goes to zero at $X=L_{\text {crit }}$.

\section{DISCUSSION AND CONCLUSION}

In this work, we did a comprehensive analysis of pure and mixed state entanglement measures, such as the entanglement entropy, mutual information, entanglement wedge cross section, and entanglement negativity, in topdown as well as bottom-up holographic confining models. For the top-down case, we considered two models that are obtained by compactifying $D 3$ and $D 4$ branes on a circle, whereas, for the bottom-up case, we considered the Einstein-Maxwell-dilaton model.

We first reproduced the known results of the entanglement entropy in these models. In particular, we reproduced the fact that, with a single strip, there are two minimal area surfaces (connected and disconnected) which exchanged dominance as the size of the subsystem is varied. This provided a phase transition in the entanglement entropy at a critical length $L_{\text {crit }}$, at which the order of the entanglement entropy changed from $\mathcal{O}\left(N^{2}\right)$ to $\mathcal{O}\left(N^{0}\right)$. We then studied the two-equal-strip entanglement phase diagram in the parameter space of $L$ and $X$ and found four distinct phases $\left\{S_{1}, S_{2}, S_{3}, S_{4}\right\}$. These four phases exchanged dominance as $L$ and $X$ are varied. The mutual information turns out to be a monotonic function of $X$ and $L$ in the $S_{2}$ and $S_{3}$ phases, and it smoothly goes to zero in the $S_{1}$ and $S_{4}$ phases. Importantly, the order of mutual information may or may not change as the critical points are crossed. The entanglement wedge cross section $E_{W}$, on the other hand, vanished discontinuously for large values of $X$ and $L$ and displayed nonanalytic behavior every time a critical point is crossed. This suggests that there can be other nontrivial length scales (apart from the natural length scale $L_{\text {crit }}$, that comes from the discontinuity in entanglement entropy) where nonanalytic entanglement structure can appear in the confining theories. Unfortunately, unlike the entanglement entropy, lattice results for $E_{W}$ are not available yet. These results therefore might be considered as genuine predictions from holography. We, moreover, tested the inequality involving mutual information and entanglement wedge cross section and found that the latter always exceeds half of the former. We further discussed the entanglement negativity with one and two intervals using the prescription suggested in Ref. [33]. A straightforward implementation of this prescription in the confined phase suggested that the entanglement negativity is proportional to the entanglement entropy and, therefore, undergoes an order change at $L_{\text {crit }}$. This suggests that, like the entanglement entropy, the entanglement negativity can also be used to probe confinement. This is again a new result, and it needs to be independently verified by lattice calculations.

We end this discussion by pointing out a few directions in which the present work can be extended. It would be 
certainly interesting to compute the entanglement negativity in confined phases using the first holographic proposal [31,32] and independently check the validity of the above mentioned results. This would be a bit nontrivial, as one first needs to compute the backreaction of cosmic brane on the spacetime geometry. On the application side, it would also be interesting to compute the entanglement wedge cross section and negativity after a global quantum quench in the confined phase and study the corresponding thermalization process, as this might provide useful information about the quark-gluon plasma formation in QCD. Another interesting direction to extend our work is to discuss the anisotropic effects on the entanglement wedge and negativity by including a background magnetic field in the lines of Refs. [119-121] and use these entanglement measures to investigate (inverse) magnetic catalysis.

\section{ACKNOWLEDGMENTS}

The work of S. M. is supported by the Department of Science and Technology, Government of India under Grant Agreement No. IFA17-PH207 (INSPIRE Faculty Award).
[1] G. Vidal, J. I. Latorre, E. Rico, and A. Kitaev, Entanglement in Quantum Critical Phenomena, Phys. Rev. Lett. 90, 227902 (2003).

[2] T. J. Osborne and M. A. Nielsen, Entanglement in a simple quantum phase transition, Phys. Rev. A 66, 032110 (2002).

[3] L. Bombelli, R. K. Koul, J. Lee, and R. D. Sorkin, A quantum source of entropy for black holes, Phys. Rev. D 34, 373 (1986).

[4] M. Srednicki, Entropy and Area, Phys. Rev. Lett. 71, 666 (1993).

[5] H-K. Lo, Classical communication cost in distributed quantum information processing-A generalization of quantum communication complexity, Phys. Rev. A 62, 012313 (2000).

[6] E. Karpov, D. Daems, and N. J. Cerf, Entanglement enhanced classical capacity of quantum communication channels with correlated noise in arbitrary dimensions, Phys. Rev. A 74, 032320 (2006).

[7] S. Ryu and T. Takayanagi, Holographic Derivation of Entanglement Entropy from AdS/CFT, Phys. Rev. Lett. 96, 181602 (2006).

[8] S. Ryu and T. Takayanagi, Aspects of holographic entanglement entropy, J. High Energy Phys. 08 (2006) 045.

[9] I. R. Klebanov, D. Kutasov, and A. Murugan, Entanglement as a probe of confinement, Nucl. Phys. B796, 274 (2008).

[10] T. Nishioka and T. Takayanagi, AdS bubbles, entropy and closed string tachyons, J. High Energy Phys. 01 (2007) 090.

[11] C. V. Johnson, Large N Phase Transitions, Finite Volume, and Entanglement Entropy, J. High Energy Phys. 03 (2014) 047.

[12] A. Dey, S. Mahapatra, and T. Sarkar, Thermodynamics and entanglement entropy with Weyl corrections, Phys. Rev. D 94, 026006 (2016).

[13] A. Dey, S. Mahapatra, and T. Sarkar, Very general holographic superconductors and entanglement thermodynamics, J. High Energy Phys. 12 (2014) 135.

[14] V. Balasubramanian, A. Bernamonti, J. de Boer, N. Copland, B. Craps, E. Keski-Vakkuri, B. Muller, A. Schafer, M. Shigemori, and W. Staessens, Holographic Thermalization, Phys. Rev. D 84, 026010 (2011).
[15] H. Liu and S. J. Suh, Entanglement Tsunami: Universal Scaling in Holographic Thermalization, Phys. Rev. Lett. 112, 011601 (2014).

[16] A. Dey, S. Mahapatra, and T. Sarkar, Holographic Thermalization with Weyl Corrections, J. High Energy Phys. 01 (2016) 088.

[17] M. Van Raamsdonk, Building up spacetime with quantum entanglement, Gen. Relativ. Gravit. 42, 2323 (2010); Int. J. Mod. Phys. D 19, 2429 (2010).

[18] V. Balasubramanian, B. D. Chowdhury, B. Czech, J. de Boer, and M. P. Heller, Bulk curves from boundary data in holography, Phys. Rev. D 89, 086004 (2014).

[19] F. Pastawski, B. Yoshida, D. Harlow, and J. Preskill, Holographic quantum error-correcting codes: Toy models for the bulk/boundary correspondence, J. High Energy Phys. 06 (2015) 149.

[20] P. Hayden, S. Nezami, X. L. Qi, N. Thomas, M. Walter, and Z. Yang, Holographic duality from random tensor networks, J. High Energy Phys. 11 (2016) 009.

[21] H. Casini, M. Huerta, and R. C. Myers, Towards a derivation of holographic entanglement entropy, J. High Energy Phys. 05 (2011) 036.

[22] A. Lewkowycz and J. Maldacena, Generalized gravitational entropy, J. High Energy Phys. 08 (2013) 090.

[23] G. Vidal and R.F. Werner, Computable measure of entanglement, Phys. Rev. A 65, 032314 (2002).

[24] R. Horodecki, P. Horodecki, M. Horodecki, and K. Horodecki, Quantum entanglement, Rev. Mod. Phys. 81, 865 (2009).

[25] B. M. Terhal, M. Horodecki, D. W. Leung, and D. P. DiVincenzo, The entanglement of purification, J. Math. Phys. (N.Y.) 43, 4286 (2002).

[26] J. Eisert and M. B. Plenio, A comparison of entanglement measures, J. Mod. Opt. 46, 145 (1999).

[27] M. Horodecki, P. Horodecki, and R. Horodecki, Separability of mixed states: Necessary and sufficient conditions, Phys. Lett. A 223, 1 (1996).

[28] A. Peres, Separability Criterion for Density Matrices, Phys. Rev. Lett. 77, 1413 (1996).

[29] T. Takayanagi and K. Umemoto, Entanglement of purification through holographic duality, Nat. Phys. 14, 573 (2018). 
[30] P. Nguyen, T. Devakul, M. G. Halbasch, M. P. Zaletel, and B. Swingle, Entanglement of purification: From spin chains to holography, J. High Energy Phys. 01 (2018) 098.

[31] J. Kudler-Flam and S. Ryu, Entanglement negativity and minimal entanglement wedge cross sections in holographic theories, Phys. Rev. D 99, 106014 (2019).

[32] Y. Kusuki, J. Kudler-Flam, and S. Ryu, Derivation of Holographic Negativity in $\mathrm{AdS}_{3} / \mathrm{CFT}_{2}$, Phys. Rev. Lett. 123, 131603 (2019).

[33] P. Chaturvedi, V. Malvimat, and G. Sengupta, Entanglement negativity, holography and black holes, Eur. Phys. J. C 78, 499 (2018).

[34] P. Chaturvedi, V. Malvimat, and G. Sengupta, Holographic quantum entanglement negativity, J. High Energy Phys. 05 (2018) 172.

[35] P. Jain, V. Malvimat, S. Mondal, and G. Sengupta, Holographic entanglement negativity conjecture for adjacent intervals in $\mathrm{AdS}_{3} / \mathrm{CFT}_{2}$, Phys. Lett. B 793, 104 (2019).

[36] P. Jain, V. Malvimat, S. Mondal, and G. Sengupta, Holographic entanglement negativity for adjacent subsystems in $\mathrm{AdS}_{d+1} / \mathrm{CFT}_{d}$, Eur. Phys. J. Plus 133, 300 (2018).

[37] P. Jain, V. Malvimat, S. Mondal, and G. Sengupta, Covariant holographic entanglement negativity for adjacent subsystems in $\mathrm{AdS}_{3} / \mathrm{CFT}_{2}$, Nucl. Phys. B945, 114683 (2019).

[38] P. Jain, V. Malvimat, S. Mondal, and G. Sengupta, Holographic entanglement negativity for conformal field theories with a conserved charge, Eur. Phys. J. C 78, 908 (2018).

[39] V. Malvimat, S. Mondal, B. Paul, and G. Sengupta, Holographic entanglement negativity for disjoint intervals in $\mathrm{AdS}_{3} / \mathrm{CFT}_{2}$, Eur. Phys. J. C 79, 191 (2019).

[40] V. Malvimat, H. Parihar, B. Paul, and G. Sengupta, Entanglement negativity in galilean conformal field theories, Phys. Rev. D 100, 026001 (2019).

[41] J. Kumar Basak, H. Parihar, B. Paul, and G. Sengupta, Holographic entanglement negativity for disjoint subsystems in $\mathrm{AdS}_{\mathrm{d}+1} / \mathrm{CFT}_{\mathrm{d}}$, arXiv:2001.10534.

[42] V. Malvimat, S. Mondal, and G. Sengupta, Time evolution of entanglement negativity from black hole interiors, J. High Energy Phys. 05 (2019) 183.

[43] S. Dutta and T. Faulkner, A canonical purification for the entanglement wedge cross-section, arXiv:1905.00577.

[44] C. Akers and P. Rath, Entanglement wedge cross sections require tripartite entanglement, J. High Energy Phys. 04 (2020) 208.

[45] P. V. Buividovich and M. I. Polikarpov, Numerical study of entanglement entropy in SU(2) lattice gauge theory, Nucl. Phys. B802, 458 (2008).

[46] P. V. Buividovich and M. I. Polikarpov, Entanglement entropy in gauge theories and the holographic principle for electric strings, Phys. Lett. B 670, 141 (2008).

[47] E. Itou, K. Nagata, Y. Nakagawa, A. Nakamura, and V. I. Zakharov, Entanglement in four-dimensional SU(3) gauge theory, Prog. Theor. Exp. Phys. 2016, 061 B01 (2016).

[48] A. Rabenstein, N. Bodendorfer, P. Buividovich, and A. Schäfer, Lattice study of Rényi entanglement entropy in $S U\left(N_{c}\right)$ lattice Yang-Mills theory with $N_{c}=2,3,4$, Phys. Rev. D 100, 034504 (2019).
[49] G. S. Ramos and M. V. T. Machado, Determination of entanglement entropy in elastic scattering using the model-independent method for hadron femtoscopy, Phys. Rev. D 102, 034019 (2020).

[50] D. Dudal and S. Mahapatra, Confining gauge theories and holographic entanglement entropy with a magnetic field, J. High Energy Phys. 04 (2017) 031.

[51] D. Dudal and S. Mahapatra, Interplay between the holographic QCD phase diagram and entanglement entropy, J. High Energy Phys. 07 (2018) 120.

[52] S. Mahapatra, Interplay between the holographic QCD phase diagram and mutual \& $n$-partite information, J. High Energy Phys. 04 (2019) 137.

[53] U. Gürsoy, M. Järvinen, G. Nijs, and J. F. Pedraza, Inverse anisotropic catalysis in holographic QCD, arXiv:1811 .11724 .

[54] U. Kol, C. Nunez, D. Schofield, J. Sonnenschein, and M. Warschawski, Confinement, phase transitions and nonlocality in the entanglement entropy, J. High Energy Phys. 06 (2014) 005.

[55] O. Ben-Ami, D. Carmi, and J. Sonnenschein, Holographic entanglement entropy of multiple strips, J. High Energy Phys. 11 (2014) 144.

[56] M. Fujita, T. Nishioka, and T. Takayanagi, Geometric Entropy and hagedorn/deconfinement transition, J. High Energy Phys. 09 (2008) 016.

[57] A. Lewkowycz, Holographic entanglement entropy and confinement, J. High Energy Phys. 05 (2012) 032.

[58] G. Georgiou and D. Zoakos, Entanglement entropy of the Klebanov-Strassler model with dynamical flavors, J. High Energy Phys. 07 (2015) 003.

[59] N. Kim, Holographic entanglement entropy of confining gauge theories with flavor, Phys. Lett. B 720, 232 (2013).

[60] M. Ghodrati, Schwinger effect and entanglement entropy in confining geometries, Phys. Rev. D 92, 065015 (2015).

[61] M. Ali-Akbari and M. Lezgi, Holographic QCD, entanglement entropy, and critical temperature, Phys. Rev. D 96, 086014 (2017).

[62] J. Knaute and B. Kämpfer, Holographic entanglement entropy in the QCD phase diagram with a critical point, Phys. Rev. D 96, 106003 (2017).

[63] M. M. Anber and B. J. Kolligs, Entanglement entropy, dualities, and deconfinement in gauge theories, arXiv:1804 .01956 .

[64] I. Y. Aref'eva, A. Patrushev, and P. Slepov, Holographic entanglement entropy in anisotropic background with confinement-deconfinement phase transition, J. High Energy Phys. 07 (2020) 043.

[65] P. Slepov, Entanglement entropy in strongly correlated systems with confinement/deconfinement phase transition and anisotropy, EPJ Web Conf. 222, 03024 (2019).

[66] P. Liu, C. Niu, and J. P. Wu, The effect of anisotropy on holographic entanglement entropy and mutual information, Phys. Lett. B 796, 155 (2019).

[67] M. Fujita, S. He, and Y. Sun, Thermodynamical property of entanglement entropy and deconfinement phase transition, arXiv:2005.01048.

[68] G. Fu, P. Liu, H. Gong, X. M. Kuang, and J. P. Wu, Informational properties for Einstein-Maxwell-Dilaton gravity, arXiv:2007.06001. 
[69] N. Jokela and A. Ponni, Notes on entanglement wedge cross sections, J. High Energy Phys. 07 (2019) 087.

[70] E. Witten, Anti-de Sitter space, thermal phase transition, and confinement in gauge theories, Adv. Theor. Math. Phys. 2, 505 (1998).

[71] D. Dudal and S. Mahapatra, Thermal entropy of a quarkantiquark pair above and below deconfinement from a dynamical holographic QCD model, Phys. Rev. D 96, 126010 (2017).

[72] P. Calabrese and J. Cardy, Entanglement entropy and conformal field theory, J. Phys. A 42, 504005 (2009).

[73] P. Hayden, M. Headrick, and A. Maloney, Holographic mutual information is monogamous, Phys. Rev. D 87, 046003 (2013).

[74] M. Headrick, Entanglement Renyi entropies in holographic theories, Phys. Rev. D 82, 126010 (2010).

[75] P. Calabrese, J. Cardy, and E. Tonni, Entanglement entropy of two disjoint intervals in conformal field theory, J. Stat. Mech. (2009) P11001.

[76] W. Fischler, A. Kundu, and S. Kundu, Holographic mutual information at finite temperature, Phys. Rev. D 87, 126012 (2013).

[77] S. Kundu and J.F. Pedraza, Aspects of holographic entanglement at finite temperature and chemical potential, J. High Energy Phys. 08 (2016) 177.

[78] H. Casini, M. Huerta, R. C. Myers, and A. Yale, Mutual information and the F-theorem, J. High Energy Phys. 10 (2015) 003.

[79] M. Alishahiha, M. R. Mohammadi Mozaffar, and M. R. Tanhayi, On the time evolution of holographic n-partite information, J. High Energy Phys. 09 (2015) 165.

[80] J. Molina-Vilaplana and P. Sodano, Holographic view on quantum correlations and mutual information between disjoint blocks of a quantum critical system, J. High Energy Phys. 10 (2011) 011.

[81] V. Balasubramanian, N. Jokela, A. Pönni, and A. V. Ramallo, Information flows in strongly coupled ABJM theory, J. High Energy Phys. 01 (2019) 232.

[82] J. Cardy, Some results on the mutual information of disjoint regions in higher dimensions, J. Phys. A 46, 285402 (2013).

[83] A. J. Larkoski, J. Thaler, and W. J. Waalewijn, Gaining (mutual) information about quark/gluon discrimination, J. High Energy Phys. 11 (2014) 129.

[84] A. Bhattacharyya, T. Takayanagi, and K. Umemoto, Entanglement of purification in free scalar field theories, J. High Energy Phys. 04 (2018) 132.

[85] A. Bhattacharyya, A. Jahn, T. Takayanagi, and K. Umemoto, Entanglement of Purification in Many Body Systems and Symmetry Breaking, Phys. Rev. Lett. 122, 201601 (2019).

[86] J. Harper and M. Headrick, Bit threads and holographic entanglement of purification, J. High Energy Phys. 08 (2019) 101.

[87] H. Hirai, K. Tamaoka, and T. Yokoya, Towards entanglement of purification for conformal field theories, Prog. Theor. Exp. Phys. 2018, 063 B03 (2018).

[88] R. Espíndola, A. Guijosa, and J. F. Pedraza, Entanglement wedge reconstruction and entanglement of purification, Eur. Phys. J. C 78, 646 (2018).
[89] K. Umemoto and Y. Zhou, Entanglement of purification for multipartite states and its holographic dual, J. High Energy Phys. 10 (2018) 152.

[90] N. Bao and I. F. Halpern, Conditional and multipartite entanglements of purification and holography, Phys. Rev. D 99, 046010 (2019).

[91] R. Q. Yang, C. Y. Zhang, and W. M. Li, Holographic entanglement of purification for thermofield double states and thermal quench, J. High Energy Phys. 01 (2019) 114.

[92] P. Caputa, M. Miyaji, T. Takayanagi, and K. Umemoto, Holographic Entanglement of Purification from Conformal Field Theories, Phys. Rev. Lett. 122, 111601 (2019).

[93] B. Amrahi, M. Ali-Akbari, and M. Asadi, Holographic entanglement of purification near a critical point, arXiv: 2004.02856.

[94] P. Liu, Y. Ling, C. Niu, and J. P. Wu, Entanglement of purification in holographic systems, J. High Energy Phys. 09 (2019) 071.

[95] Y. Nomura, P. Rath, and N. Salzetta, Pulling the boundary into the bulk, Phys. Rev. D 98, 026010 (2018).

[96] K. Babaei Velni, M. R. Mohammadi Mozaffar, and M. H. Vahidinia, Some aspects of entanglement wedge crosssection, J. High Energy Phys. 05 (2019) 200.

[97] P. Liu and J. P. Wu, Mixed state entanglement and thermal phase transitions, arXiv:2009.01529.

[98] P. Calabrese, J. Cardy, and E. Tonni, Entanglement Negativity in Quantum Field Theory, Phys. Rev. Lett. 109, 130502 (2012).

[99] P. Calabrese, J. Cardy, and E. Tonni, Entanglement negativity in extended systems: A field theoretical approach, J. Stat. Mech. (2013) P02008.

[100] V. Alba, Entanglement negativity and conformal field theory: A Monte Carlo study, J. Stat. Mech. (2013) P05013.

[101] P. Calabrese, L. Tagliacozzo, and E. Tonni, Entanglement negativity in the critical Ising chain, J. Stat. Mech. (2013) P05002.

[102] C. M. Chung, V. Alba, L. Bonnes, P. Chen, and A. M. L auchli, Entanglement negativity via the replica trick: A quantum Monte Carlo approach, Phys. Rev. B 90, 064401 (2014).

[103] P. Ruggiero, V. Alba, and P. Calabrese, Entanglement negativity in random spin chains, Phys. Rev. B 94, 035152 (2016).

[104] P. Ruggiero, V. Alba, and P. Calabrese, Negativity spectrum of one-dimensional conformal field theories, Phys. Rev. B 94, 195121 (2016).

[105] A. Coser, E. Tonni, and P. Calabrese, Entanglement negativity after a global quantum quench, J. Stat. Mech. (2014) P12017.

[106] M. Hoogeveen and B. Doyon, Entanglement negativity and entropy in non-equilibrium conformal field theory, Nucl. Phys. B898, 78 (2015).

[107] O. Blondeau-Fournier, O. A. Castro-Alvaredo, and B. Doyon, Universal scaling of the logarithmic negativity in massive quantum field theory, J. Phys. A 49, 125401 (2016).

[108] C. Castelnovo, Negativity and topological order in the toric code, Phys. Rev. A 88, 042319 (2013).

[109] Y.A. Lee and G. Vidal, Entanglement negativity and topological order, Phys. Rev. A 88, 042318 (2013). 
[110] V. Eisler and Z. Zimboras, Entanglement negativity in twodimensional free lattice models, New J. Phys. 16, 123020 (2015).

[111] X. Wen, P. Y. Chang, and S. Ryu, Entanglement negativity after a local quantum quench in conformal field theories, Phys. Rev. B 92, 075109 (2015).

[112] X. Wen, P. Y. Chang, and S. Ryu, Topological entanglement negativity in Chern-Simons theories, J. High Energy Phys. 09 (2016) 012.

[113] X. Wen, S. Matsuura, and S. Ryu, Edge theory approach to topological entanglement entropy, mutual information and entanglement negativity in Chern-Simons theories, Phys. Rev. B 93, 245140 (2016).

[114] M. Rangamani and M. Rota, Comments on entanglement negativity in holographic field theories, J. High Energy Phys. 10 (2014) 060.

[115] N. Jokela and J. G. Subils, Is entanglement a probe of confinement?, arXiv:2010.09392.

[116] I. R. Klebanov and M. J. Strassler, Supergravity and a confining gauge theory: Duality cascades and chi SB resolution of naked singularities, J. High Energy Phys. 08 (2000) 052.

[117] S. Mahapatra and P. Roy, On the time dependence of holographic complexity in a dynamical Einstein-dilaton model, J. High Energy Phys. 11 (2018) 138.

[118] S. Mahapatra, S. Priyadarshinee, G. N. Reddy, and B. Shukla, Exact topological charged hairy black holes in AdS space in D-dimensions, Phys. Rev. D 102, 024042 (2020).

[119] H. Bohra, D. Dudal, A. Hajilou, and S. Mahapatra, Anisotropic string tensions and inversely magnetic catalyzed deconfinement from a dynamical AdS/QCD model, Phys. Lett. B 801, 135184 (2020).

[120] H. Bohra, D. Dudal, A. Hajilou, and S. Mahapatra, Chiral transition in the probe approximation from an Einstein-Maxwell-dilaton gravity model, arXiv:2010 .04578 .

[121] I. Aref'eva and K. Rannu, Holographic anisotropic background with confinement-deconfinement phase transition, J. High Energy Phys. 05 (2018) 206. 